\title{
SECOND DERIVATIVES ESTIMATE OF SUITABLE SOLUTIONS TO THE 3D NAVIER-STOKES EQUATIONS
}

\author{
ALEXIS VASSEUR AND JINCHENG YANG
}

\begin{abstract}
We study the second spatial derivatives of suitable weak solutions to the incompressible Navier-Stokes equations in dimension three. We show that it is locally $L^{\frac{4}{3}, q}$ for any $q>\frac{4}{3}$, which improves from the current result $L^{\frac{4}{3}, \infty}$. Similar improvements in Lorentz space are also obtained for higher derivatives of the vorticity for smooth solutions. We use a blow-up technique to obtain nonlinear bounds compatible with the scaling. The local study works on the vorticity equation and uses De Giorgi iteration. In this local study, we can obtain any regularity of the vorticity without any a priori knowledge of the pressure. The local-to-global step uses a recently constructed maximal function for transport equations.

Keywords. Navier-Stokes Equation, Partial Regularity, Blow-up Techniques, De Giorgi Method, Maximal Function, Lorentz Space.
\end{abstract}

\section{Contents}

1. Introduction

2. Preliminary

2.1. Maximal Function associated with Skewed Cylinders

2.2. Lorentz Space

2.3. Helmholtz decomposition

3. Proof of the Main Results

4. Local Study: Part One, Initial Energy 12

4.1. Equations of $\mathrm{v} \quad 13$

4.2. Energy Estimate 16

4.3. Proof of Proposition $4.1 \quad 17$

5. Local Study: Part Two, De Giorgi Iteration 19

5.1. Highest Order Nonlinear Term 21

5.2. Lower Order Terms 23

5.3. W Terms 24

5.4. Proof of Proposition $5.1 \quad 26$

6. Local Study: Part Three, More Regularity 26

6.1. Bound Vorticity in the Energy Space 27

6.2. Bound Higher Derivatives in the Energy Space 30

6.3. Proof of the Local Theorem 34

Appendix A. Suitability of Solutions

References

Date: October 1, 2020.

2020 Mathematics Subject Classification. 76D05, 35Q30.

Acknowledgement. A. Vasseur was partially supported by the NSF grant: DMS 1614918. 


\section{INTRODUCTION}

We study the three dimensional incompressible Navier-Stokes equations,

$$
\partial_{t} u+u \cdot \nabla u+\nabla P=\Delta u, \quad \operatorname{div} u=0 .
$$

Here $u:(0, T) \times \mathbb{R}^{3} \rightarrow \mathbb{R}^{3}$ and $P:(0, T) \times \mathbb{R}^{3} \rightarrow \mathbb{R}$ represent the velocity field and the pressure field of a fluid in $\mathbb{R}^{3}$, within a finite or infinite timespan of length $T$. Initial condition

$$
u(0, \cdot)=u_{0} \in L^{2}\left(\mathbb{R}^{3}\right)
$$

is given by a divergence-free velocity profile $u_{0}$ of finite energy.

Leray ([Ler34) and Hopf ([Hop51) proved the existence of weak solutions for all time. They constructed solutions $u \in C_{w}\left(0, \infty ; L^{2}\left(\mathbb{R}^{3}\right)\right) \cap L^{2}\left(0, \infty ; \dot{H}^{1}\left(\mathbb{R}^{3}\right)\right)$ corresponding to each aforementioned initial value, and satisfying (1.1) in the sense of distribution. A weak solution is called a Leray-Hopf solution if it satisfies energy inequality

$$
\frac{1}{2}\|u(t)\|_{L^{2}\left(\mathbb{R}^{3}\right)}^{2}+\|\nabla u\|_{L^{2}\left((0, t) \times \mathbb{R}^{3}\right)}^{2} \leq \frac{1}{2}\left\|u_{0}\right\|_{L^{2}\left(\mathbb{R}^{3}\right)}^{2}
$$

for every $t>0$. Since then, much work has been developed in regard to the uniqueness and regularity of weak solutions. Nonuniqueness of weak solutions was proven very recently by Buckmaster and Vicol ([BV19]) using convex integration scheme. However, the question of the uniqueness of Leray-Hopf solutions still remains open. The uniqueness is related with the regularity of solutions by the Ladyženskaya-Prodi-Serrin criteria ([KL57, Pro59, Ser62, Ser63, FJR72]): if the velocity belongs to any space interpolating $L_{t}^{2} L_{x}^{\infty}$ and $L_{t}^{\infty} L_{x}^{3}$ then it is actually smooth, hence unique. The endpoint case $L_{t}^{\infty} L_{x}^{3}$ comes much later by Iskauriaza, Serëgin and Shverak [SS03. These spaces require $\frac{1}{6}$ higher spatial integrability than the energy space provides, which is $\mathcal{E}=L_{t}^{\infty} L_{x}^{2} \cap L_{t}^{2} \dot{H}_{x}^{1}$.

At the level of energy space, Scheffer ( Sch76, Sch77, Sch78, Sch80]) began to study the partial regularity for a class of Leray-Hopf solutions, called suitable weak solutions. These solutions exist globally and satisfy the following local energy inequality,

$$
\partial_{t} \frac{|u|^{2}}{2}+\operatorname{div}\left(u\left(\frac{|u|^{2}}{2}+P\right)\right)+|\nabla u|^{2} \leq \Delta \frac{|u|^{2}}{2} .
$$

Scheffer showed the singular set, at which the solution is unbounded nearby, has time-space Hausdorff dimension at most $\frac{5}{3}$. This result was later improved by Caffarelli, Kohn and Nirenberg in [CKN82 (see also [Lin98, Vas07]), where they showed the 1-dimensional Hausdorff measure of the singular set is zero. We will investigate the regularity of suitable weak solutions. In the periodic setting, Constantin (Con90) constructed suitable weak solutions whose second derivatives have space-time integrability $L^{\frac{4}{3}-\varepsilon}$ for any $\varepsilon>0$, provided the initial vorticities are bounded measures. This was improved by Lions (Lio96) to a slightly better space $L^{\frac{4}{3}, \infty}$, a Lorentz space which corresponds to weak $L^{\frac{4}{3}}$ space. These estimates are extended to higher derivatives of smooth solutions by one of the authors and Choi ([Vas10, CV14]) using blow-up arguments: $L_{\text {loc }}^{p, \infty}$ space-time boundedness for $(-\Delta)^{\frac{\alpha}{2}} \nabla^{n} u$, where $p=\frac{4}{n+\alpha+1}, n \geq 1,0 \leq \alpha<2$. They also constructed suitable weak solutions satisfying these bounds for $n+\alpha<3$. 
The aim of this paper is to improve these regularity results in Lorentz space. The main result is the following. Note that the estimate does not rely on the size of the pressure.

Theorem 1.1. Suppose we have a smooth solution $u$ to the Navier-Stokes equations in $(0, T) \times \mathbb{R}^{3}$ for some $0<T \leq \infty$ with smooth divergence free initial data $u_{0} \in L^{2}$. Then for any integer $n \geq 0$, for any real number $q>1$, the vorticity $\omega=\operatorname{curl} u$ satisfies

$$
\left\|\left|\nabla^{n} \omega\right|^{\frac{4}{n+2}} 1_{\left\{\left|\nabla^{n} \omega\right|^{\frac{4}{n+2}}>C_{n} t^{-2}\right\}}\right\|_{L^{1, q}\left((0, T) \times \mathbb{R}^{3}\right)} \leq C_{q, n}\left\|u_{0}\right\|_{L^{2}}^{2}
$$

for some constant $C_{n}$ depending on $n$ and $C_{q, n}$ depending only on $q$ and $n$, uniform in $T$. The above estimate (1.2) also holds for suitable weak solutions with only $L^{2}$ divergence free initial data in the case $n=1$.

This theorem gives the following improvement on the second derivatives.

Corollary 1.2. Let $u$ be a suitable weak solution in $(0, \infty) \times \mathbb{R}^{3}$ with initial data $u_{0} \in L^{2}$. Then for any $q>\frac{4}{3}, K \subset \subset(0, \infty) \times \mathbb{R}^{3}$, there exists a constant $C_{q, K}$ depending on $q$ and $K$ such that the following holds,

$$
\left\|\nabla^{2} u\right\|_{L^{\frac{4}{3}, q}(K)} \leq C_{q, K}\left(\left\|u_{0}\right\|_{L^{2}}^{\frac{3}{2}}+1\right) .
$$

Let us explain the main ideas of the proof. Similar as previous work on higher derivatives, the proof is also based on blow-up techniques. In particular, we blow up the equation along a trajectory, using the scaling symmetry and the Galilean invariance of the Navier-Stokes equations. That is, if we fix an initial time $t_{0}$ and move the frame of reference along some $X(t)$, and zoom in into $\varepsilon$ scale, then it is easy to verify that $\tilde{u}(s, y), \tilde{P}(s, y)$ defined by

$$
\begin{aligned}
& \frac{1}{\varepsilon} \tilde{u}\left(\frac{t-t_{0}}{\varepsilon^{2}}, \frac{x-X(t)}{\varepsilon}\right):=u(t, x)-\dot{X}(t) \\
& \frac{1}{\varepsilon^{2}} \tilde{P}\left(\frac{t-t_{0}}{\varepsilon^{2}}, \frac{x-X(t)}{\varepsilon}\right):=P(t, x)+x \cdot \ddot{X}(t)
\end{aligned}
$$

also satisfy the Navier-Stokes equation

$$
\partial_{s} \tilde{u}+\tilde{u} \cdot \nabla \tilde{u}+\nabla \tilde{P}=\Delta \tilde{u}, \quad \operatorname{div} \tilde{u}=0 .
$$

We develop the following local theorem for $\tilde{u}$ and $\tilde{P}$. Note that it needs nothing from the pressure. Denote $B_{r} \subset \mathbb{R}^{3}$ to be a ball centered at the origin with radius $r$, and $Q_{r}=\left(-r^{2}, 0\right) \times B_{r} \subset \mathbb{R}^{4}$ to be a space-time cylinder.

Theorem 1.3 (Local Theorem). There exists a universal constant $\eta_{1}>0$, such that for any suitable weak solution $u$ to the Navier-Stokes equations in $(-4,0) \times \mathbb{R}^{3}$ satisfying

$$
\begin{aligned}
& \int_{B_{1}} u(t, x) \phi(x) \mathrm{d} x=0 \quad \text { a.e. } t \in(-4,0), \\
& \|\nabla u\|_{L_{t}^{p_{1}} L_{x}^{q_{1}}\left(Q_{2}\right)}+\|\omega\|_{L_{t}^{p_{2}} L_{x}^{q_{2}}\left(Q_{2}\right)} \leq \eta_{1},
\end{aligned}
$$

where $\phi \in C_{c}^{\infty}\left(B_{1}\right)$ is a non-negative function with $\int \phi=1, \omega=$ curl $u$ is the vorticity, $\frac{4}{3} \leq p_{1} \leq \infty, 1 \leq p_{2} \leq \infty, 1 \leq q_{1}, q_{2}<3$ satisfying

$$
\frac{1}{p_{1}}+\frac{1}{p_{2}}<1, \quad \frac{1}{q_{1}}+\frac{1}{q_{2}} \leq \frac{7}{6},
$$


then for any integer $n \geq 0$, we have

$$
\left\|\nabla^{n} \omega\right\|_{L^{\infty}\left(Q_{8-n-2}\right)} \leq C_{n}
$$

for some constant $C_{n}$ depending only on $n$.

Let us illustrate the ideas of how to go from this local theorem towards the main result. We want to choose a "pivot quantity", blow up near a point, and use this quantity to control $\nabla^{n} \omega$. When we patch the local results together, we will obtain a nonlinear bound with the same scaling as the pivot quantity, so we want the pivot quantity to have the best possible scaling. The ideal pivot quantities would be $\int|\nabla u|^{2} \mathrm{~d} x \mathrm{~d} t$ and $\int\left|\nabla^{2} P\right| \mathrm{d} x \mathrm{~d} t . \int|u|^{\frac{10}{3}} \mathrm{~d} x \mathrm{~d} t$ has a worse scaling and should not be used. However, we still need to control the flux in the local theorem, so we want to take out the mean velocity and control $u$ by $\nabla u$ using Poincaré's inequality.

In order to take out the mean velocity, we choose $X(t)$ to be the trajectory of the mollified flow so that (1.4) can be realized. Notice that a cylinder $Q_{r}$ in the local $(s, y)$ coordinate will be transformed into a "skewed cylinder" growing along $X(t)$ in the global $(t, x)$ coordinate. One of the authors recently constructed a maximal function $\mathcal{M}_{\mathcal{Q}}$ associated with these cylinders ([Yan20]), which serves as a bridge between the local theorem and the global result, and is one of the main reasons for the improvement in this paper. The idea is, if locally the vorticity gradient can be controlled in $L^{\infty}$ by the integral of something in the skewed cylinder, and the integral in a skewed cylinder can be controlled by the maximal function $\mathcal{M}_{\mathcal{Q}}$, then vorticity gradient is pointwisely bounded by the maximal function.

If one uses $\int|\nabla u|^{2} \mathrm{~d} x \mathrm{~d} t$ and $\int\left|\nabla^{2} P\right| \mathrm{d} x \mathrm{~d} t$ as the pivot quantity, then unfortunately the best possible outcome would just be an $L^{1, \infty}$ bound, as obtained in Yan20. The reason is, the maximal function is bounded on $L^{p}$ for $p>1$, but for $p=1$ it is only bounded from $L^{1}$ to $L^{1, \infty}$. Unfortunately $|\nabla u|^{2}$ and $\left|\nabla^{2} P\right|$ are both $L^{1}$ quantities, so $\mathcal{M}_{\mathcal{Q}}\left(|\nabla u|^{2}+\left|\nabla^{2} P\right|\right)$ is only $L^{1, \infty}$. We need two things to improve from $L^{1, \infty}$ : replace $\int|\nabla u|^{2}$ by $\int|\nabla u|^{p}$, and drop the pressure $\nabla^{2} P$.

Suppose we could use $\left(\int|\nabla u|^{p} \mathrm{~d} x \mathrm{~d} t\right)^{\frac{2}{p}}$ as the pivot quantity for some $p<2$, then we can majorize it by $\mathcal{M}_{\mathcal{Q}}\left(|\nabla u|^{p}\right)^{\frac{2}{p}} \in L^{1}$, since $\frac{2}{p}>1$ and $\mathcal{M}_{\mathcal{Q}}$ is bounded in $L^{\frac{2}{p}}$. However, this poses significant difficulties in the local theorem. The nonlinear term $u \cdot \nabla u$ is quadratic, and if we only have a subquadratic integrability to begin with, we cannot treat this quadratic transport term as a source term because it is not integrable. Observe that what we lack of is the temporal integrability rather than the spatial one: if $p$ is slightly smaller than two, than $u \cdot \nabla u$ is still $L^{\frac{3}{2}-}$ in space, but $L^{1-}$ in time. To overcome this difficulty, we write $u \cdot \nabla u$ as $\omega \times u$ up to a gradient term, and put $L_{t}^{2-} L_{x}^{6-}$ on $u$ and $L_{t}^{2+} L_{x}^{2-}$ on $\omega$. We compensate the lower integrability term by pairing with a higher integrability term to make $\omega \times u$ integrable. $L_{t}^{2+} L_{x}^{2-}$ of $\omega$ can be interpolated between $L_{t}^{2-} L_{x}^{2-}$ and $L_{t}^{\infty} L_{x}^{1}$, while the latter is controlled by $L_{t, x}^{2}$ of $\nabla u$. Since $L_{t}^{2+} L_{x}^{2-}$ is closer to $L_{t}^{2-} L_{x}^{2-}$ than to $L_{t}^{\infty} L_{x}^{1}$, the pivot quantity that we use is actually $\delta^{-\nu}\|\nabla u\|_{L^{p}}^{2}+\delta\|\nabla u\|_{L^{2}}^{2}$ for $\nu$ close to 0 . By using more subquadratic integrability and a tiny bit of the quadratic one, we can complete the task by interpolation. That is why we obtain $L^{1, q}$ in the end: it interpolates $L^{1}$ bound from $\|\nabla u\|_{L^{p}}$ and $L^{1, \infty}$ bound from $\|\nabla u\|_{L^{2}}$. Unfortunately we still miss the endpoint $L^{1}$.

The second task is more subtle and technical. Without any information on the pressure, we don't have any control on the nonlocal effect. However, the role of the 
pressure is not important at the vorticity level: if we take the curl of the NavierStokes equation, the pressure will disappear and we are left with the vorticity equation involving only local quantities:

$$
\partial_{t} \omega+u \cdot \nabla \omega-\omega \cdot \nabla u=\Delta \omega .
$$

Inspired by Chamorro, Lemarié-Rieusset and Mayoufi ([CLRM18]), we introduce a new velocity variable $v=-\operatorname{curl} \varphi^{\sharp} \Delta^{-1} \varphi \omega$ using only local information of vorticity ( $\varphi$ and $\varphi^{\sharp}$ are spatial cut-off functions), and this helps us to prove the local theorem. This is another main reason for the improvement in this paper. Consequently, the bounds we obtain in the end is on the vorticity $\omega$ rather than on the velocity $u$.

This paper is organized as follows. In the preliminary Section 2 we introduce the analysis tools to the reader. We show how to rigorously derive the main results from the local theorem in Section 3, and then deal with technicalities of the local theorem in the later sections. The proof of the local theorem consists of three parts. Section 4 introduces the new variables $v$, and shows the smallness of $v$ in the energy space. Then we use De Giorgi iteration argument in Section 5 to prove boundedness of $v$. Finally, we inductively bound $\omega$ and all its higher derivatives in Section 6 .

\section{Preliminary}

In this section, we introduce a few tools that we are going to use in the paper, including the maximal function, Lorentz space, and Helmholtz decomposition.

2.1. Maximal Function associated with Skewed Cylinders. This is recently developed for incompressible flows in Yan20. We quote useful results here without proof.

Suppose $u \in L^{p}\left(0, T ; \dot{W}^{1, p}\left(\mathbb{R}^{3} ; \mathbb{R}^{3}\right)\right)$ is a vector field in $\mathbb{R}^{3}$. Fix $\phi \in C_{c}^{\infty}\left(B_{1}\right)$ to be a nonnegative function with $\int \phi=1$ through out the paper. For $\varepsilon>0$ define $\phi_{\varepsilon}(x)=\varepsilon^{-3} \phi(-x / \varepsilon)$, and let $u_{\varepsilon}(t, \cdot)=u(t, \cdot) * \phi_{\varepsilon}$ be the mollified velocity. For a fixed $(t, x)$ we let $X(s)$ solve the following initial value problem,

$$
\left\{\begin{array}{l}
\dot{X}(s)=u_{\varepsilon}(s, X(s)), \\
X(t)=x
\end{array}\right.
$$

The skewed parabolic cylinder $Q_{\varepsilon}(t, x)$ is then defined to be

$$
Q_{\varepsilon}(t, x):=\left\{\left(t+\varepsilon^{2} s, X(t)+\varepsilon y\right):-9 \leq s \leq 0, y \in B_{3}\right\} .
$$

We use $\mathcal{M}$ to denote the spatial Hardy-Littlewood maximal function, which is defined by

$$
\mathcal{M}(f)(t, x)=\sup _{r>0} f_{B_{r}(x)}|f(t, y)| \mathrm{d} y .
$$

Then we construct the space-time maximal function adapted to the flow.

Theorem 2.1 (Q $\mathcal{Q}$-Maximal Function). There exists a universal constant $\eta_{0}$ such that the following is true. We say $Q_{\varepsilon}(t, x)$ is admissible if $Q_{\varepsilon}(t, x) \subset(0, T) \times \mathbb{R}^{3}$ and

$$
\varepsilon^{2} f_{Q_{\varepsilon}(t, x)} \mathcal{M}(|\nabla u|) \mathrm{d} x \mathrm{~d} t \leq \eta_{0}
$$


Define the maximal function

$$
\mathcal{M}_{\mathcal{Q}}(f)(t, x):=\sup _{\varepsilon>0}\left\{f_{Q_{\varepsilon}(t, x)}|f(s, y)| \mathrm{d} s \mathrm{~d} y: Q_{\varepsilon}(t, x) \text { is admissible }\right\} .
$$

If $u$ is divergence free and $\mathcal{M}(|\nabla u|) \in L^{q}$ for some $1 \leq q \leq \infty$, then $\mathcal{M}_{\mathcal{Q}}$ is bounded from $L^{1}\left((0, T) \times \mathbb{R}^{3}\right)$ to $L^{1, \infty}\left((0, T) \times \mathbb{R}^{3}\right)$ and from $L^{p}\left((0, T) \times \mathbb{R}^{3}\right)$ to itself for any $p>1$ with norm depending on $p$.

An important consequence of the weak type $(1,1)$ bound of the Hardy-Littlewood maximal function is the Lebesgue differentiation theorem in $\mathbb{R}^{n}$. Similarly, we can use the $\mathcal{Q}$-maximal function to prove the $\mathcal{Q}$-Lebesgue differentiation theorem.

Theorem 2.2 (Q $\mathcal{Q}$-Lebesgue Differentiation Theorem). Let $f \in L_{\text {loc }}^{1}\left((0, T) \times \mathbb{R}^{3}\right)$. Then for almost every $(t, x) \in(0, T) \times \mathbb{R}^{3}$,

$$
\lim _{\varepsilon \rightarrow 0} f_{Q_{\varepsilon}(t, x)}|f(s, y)-f(t, x)| \mathrm{d} s \mathrm{~d} y=0 .
$$

In this case we say $(t, x)$ is a $\mathcal{Q}$-Lebesgue point of $f$.

2.2. Lorentz Space. Let $(X, \mu)$ be a measure space. Recall that for a measurable function $f$, its decreasing rearrangement is defined as

$$
f^{*}(\lambda):=\inf \{\alpha>0: \mu(\{|f|>\alpha\})<\lambda\}, \quad \lambda \geq 0 .
$$

For $0<p<\infty, 0<q \leq \infty$, Lorentz space $L^{p, q}(X)$ is defined as the set of functions $f$ for which

$$
\|f\|_{L^{p, q}(X)}:=\left\|t^{\frac{1}{p}} f^{*}\right\|_{L^{q}\left(\frac{\mathrm{d} t}{t}\right)}<\infty .
$$

Now we introduct the interpolation lemma for Lorentz spaces.

Lemma 2.3 (Interpolation of Lorentz Spaces). Let $\nu>0$ be a fixed positive number. Assume $f_{0} \in L^{p_{0}, q_{0}}, f_{1} \in L^{p_{1}, q_{1}}$, where $0<p_{0}, p_{1}<\infty, 0<q_{0}, q_{1} \leq \infty$. If $f$ is a measurable function satisfying

$$
2|f| \leq \delta f_{0}+\delta^{-\nu} f_{1} \quad \forall \delta>0
$$

then $f \in L^{p, q}$, where

$$
\frac{1}{p}=\frac{\nu}{1+\nu} \frac{1}{p_{0}}+\frac{1}{1+\nu} \frac{1}{p_{1}}, \quad \frac{1}{q}=\frac{\nu}{1+\nu} \frac{1}{q_{0}}+\frac{1}{1+\nu} \frac{1}{q_{1}} .
$$

Proof. Upon on decreasing rearrangement, we may assume $f, f_{0}, f_{1}$ are nonnegative decreasing functions on $[0, \infty)$. Set $\theta=\frac{1}{1+\nu}, \delta=f_{0}^{-\theta} f_{1}^{\theta}$, then

$$
2|f| \leq f_{0}^{-\theta} f_{1}^{\theta} f_{0}+f_{0}^{\nu \theta} f_{1}^{-\nu \theta} f_{1}=2 f_{0}^{1-\theta} f_{1}^{\theta} .
$$

Then

$$
\begin{aligned}
\|f\|_{L^{p, q}} & =\left\|\lambda^{\frac{1}{p}-\frac{1}{q}} f(\lambda)\right\|_{L^{q}} \\
& \leq\left\|\lambda^{\frac{1-\theta}{p_{0}}-\frac{1-\theta}{q_{0}}} f_{0}^{1-\theta}(\lambda) \cdot \lambda^{\frac{\theta}{p_{1}}-\frac{\theta}{q_{1}}} f_{1}^{\theta}(\lambda)\right\|_{L^{q}} \\
& \leq\left\|\lambda^{\frac{1-\theta}{p_{0}}-\frac{1-\theta}{q_{0}}} f_{0}^{1-\theta}(\lambda)\right\|_{L^{\frac{q_{0}}{1-\theta}}}\left\|\lambda^{\frac{\theta}{p_{1}}-\frac{\theta}{q_{1}}} f_{1}^{\theta}(\lambda)\right\|_{L^{\frac{q_{1}}{\theta}}} \\
& =\left\|\lambda^{\frac{1}{p_{0}}-\frac{1}{q_{0}}} f_{0}\right\|_{L^{q_{0}}}^{1-\theta}\left\|\lambda^{\frac{1}{p_{1}}-\frac{1}{q_{1}}} f_{1}\right\|_{L^{q_{1}}}^{\theta} \\
& =\left\|f_{0}\right\|_{L^{p_{0}, q_{0}}}^{1-\theta}\left\|f_{1}\right\|_{L^{p_{1}, q_{1}}}^{\theta} .
\end{aligned}
$$


We would also like to mention that Riesz transform is bounded on Lorentz space. The proof can be found in [CF07]. See [Saw90] for general Lorentz spaces.

Lemma 2.4. For $1<p<\infty, 1 \leq q \leq \infty, R_{i j}=\partial_{i} \partial_{j} \Delta^{-1}$ is a bounded linear operator from $L^{p, q}\left(\mathbb{R}^{n}\right)$ to itself. As a spatial operator, it is also bounded in timespace from $L^{p, q}\left((0, T) \times \mathbb{R}^{n}\right)$ to itself.

2.3. Helmholtz decomposition. First recall two vector calculus identities:

$$
\begin{aligned}
\nabla(u \cdot v) & =(u \cdot \nabla) v+(v \cdot \nabla) u+u \times \operatorname{curl} v+v \times \operatorname{curl} u, \\
\operatorname{curl}(u \times v) & =u \operatorname{div} v-v \operatorname{div} u+(v \cdot \nabla) u-(u \cdot \nabla) v .
\end{aligned}
$$

For operators $A$ and $B$, denote $[A, B]=A B-B A$ to be their commutator. Define $\mathbb{P}_{\text {curl }}=-$ curlcurl $\Delta^{-1}$ and $\mathbb{P}_{\nabla}=\nabla \Delta^{-1} \operatorname{div}=\mathrm{Id}-\mathbb{P}_{\text {curl }}$ to be the Helmholtz decomposition. Then we compute the following commutators.

$$
\begin{aligned}
& {[\varphi, \operatorname{curl}] u=}-\nabla \varphi \times u \\
& {[\varphi, \Delta] u=- } 2 \nabla \varphi \cdot \nabla u-(\Delta \varphi) u=-2 \operatorname{div}(\nabla \varphi \otimes u)+(\Delta \varphi) u \\
& {\left[\varphi, \Delta^{-1}\right] u=\Delta^{-1}\left\{2 \nabla \varphi \cdot \nabla \Delta^{-1} u+(\Delta \varphi) \Delta^{-1} u\right\} } \\
& {\left[\varphi, \mathbb{P}_{\text {curl }}\right] u=\nabla \varphi \times \operatorname{curl} \Delta^{-1} u+\nabla \varphi \operatorname{div} \Delta^{-1} u-\Delta^{-1} u \Delta \varphi } \\
& \\
&+\left(\Delta^{-1} u \cdot \nabla\right) \nabla \varphi-(\nabla \varphi \cdot \nabla) \Delta^{-1} u \\
&+ \\
&+\mathbb{P}_{\text {curl }}\left\{2 \nabla \varphi \cdot \nabla \Delta^{-1} u+(\Delta \varphi) \Delta^{-1} u\right\}
\end{aligned}
$$

The first two are straightforward. The third uses

$$
\left[\varphi, \Delta^{-1}\right]=-\Delta^{-1}[\varphi, \Delta] \Delta^{-1}
$$

and the last one is because

$$
\begin{aligned}
{\left[\varphi, \mathbb{P}_{\text {curl }}\right]=} & {\left[\varphi,-\operatorname{curl} \operatorname{curl} \Delta^{-1}\right] } \\
= & -[\varphi, \operatorname{curl}] \operatorname{curl} \Delta^{-1}-\operatorname{curl}[\varphi, \operatorname{curl}] \Delta^{-1}-\operatorname{curl} \operatorname{curl}\left[\varphi, \Delta^{-1}\right], \\
{\left[\varphi, \mathbb{P}_{\text {curl }}\right] u=} & \nabla \varphi \times \operatorname{curl} \Delta^{-1} u+\operatorname{curl}\left(\nabla \varphi \times \Delta^{-1} u\right) \\
& \quad-\operatorname{curl} \operatorname{curl} \Delta^{-1}\left\{2 \nabla \varphi \cdot \nabla \Delta^{-1} u+(\Delta \varphi) \Delta^{-1} u\right\},
\end{aligned}
$$

and we can expand $\operatorname{curl}\left(\nabla \varphi \times \Delta^{-1} u\right)$ by (2.4).

Lemma 2.5. $\partial_{i}\left[\varphi, \mathbb{P}_{\text {curl }}\right]$ and $\left[\varphi, \mathbb{P}_{\text {curl }}\right] \partial_{i}$ are both bounded linear operator from $L^{p}$ to $L^{p}$ for any $1<p<\infty$, i.e.

$$
\left\|\partial_{i}\left[\varphi, \mathbb{P}_{\text {curl }}\right] u\right\|_{L^{p}}+\left\|\left[\varphi, \mathbb{P}_{\text {curl }}\right] \partial_{i} u\right\|_{L^{p}} \leq C_{p, \varphi}\|u\|_{L^{p}} .
$$

Proof. First, we observe that by Jacobi identity $\left[\varphi, \mathbb{P}_{\text {curl }}\right] \partial_{i}$ and $\partial_{i}\left[\varphi, \mathbb{P}_{\text {curl }}\right]$ differ by

$$
\left[\left[\varphi, \mathbb{P}_{\text {curl }}\right], \partial_{i}\right]=\left[\varphi,\left[\mathbb{P}_{\text {curl }}, \partial_{i}\right]\right]-\left[\mathbb{P}_{\text {curl }},\left[\varphi, \partial_{i}\right]\right]=0-\left[\mathbb{P}_{\text {curl }}, \partial_{i} \varphi\right]
$$

which is bounded from $L^{p}$ to $L^{p}$ for any $p$, because both $\mathbb{P}_{\text {curl }}$ and multiplication by $\partial_{i} \varphi$ are bounded from $L^{p}$ to $L^{p}$, so we can complete the proof by duality. For $1<p<3$, set $\frac{1}{p^{*}}=\frac{1}{p}-\frac{1}{3}$, from (2.8) we can see

$$
\begin{aligned}
\left\|\left[\varphi, \mathbb{P}_{\text {curl }}\right] \partial_{i} u\right\|_{L^{p}} & \lesssim\left\|\nabla \Delta^{-1} \partial_{i} u\right\|_{L^{p}\left(\mathbb{R}^{3}\right)}+C_{p, \varphi}\left\|\Delta^{-1} \partial_{i} u\right\|_{L^{p}(\operatorname{supp} \varphi)} \\
& \lesssim\|u\|_{L^{p}\left(\mathbb{R}^{3}\right)}+C_{p, \varphi}\left\|\partial_{i} \Delta^{-1} u\right\|_{L^{p^{*}}(\operatorname{supp} \varphi)} \\
& \leq C\|u\|_{L^{p}\left(\mathbb{R}^{3}\right)} .
\end{aligned}
$$


For $\frac{3}{2}<p<\infty$, set $1-\frac{1}{p}=\frac{1}{q}=\frac{1}{q^{*}}+\frac{1}{3}$, then $1<p, q, q^{*}<\infty$. Take any $u \in L^{p}\left(\mathbb{R}^{3}\right)$ and any vector field $v \in L^{q}\left(\mathbb{R}^{3}\right)$,

$$
\begin{aligned}
\int \partial_{i}\left[\varphi, \mathbb{P}_{\text {curl }}\right] u \cdot v \mathrm{~d} x & =-\int\left[\varphi, \mathbb{P}_{\text {curl }}\right] u \cdot \partial_{i} v \mathrm{~d} x \\
& =\int u \cdot\left[\varphi, \mathbb{P}_{\text {curl }}\right] \partial_{i} v \mathrm{~d} x \\
& \leq\|u\|_{L^{p}\left(\mathbb{R}^{3}\right)}\left(\|v\|_{L^{q}\left(\mathbb{R}^{3}\right)}+\left\|\partial_{i} \Delta^{-1} v\right\|_{L^{q}(\operatorname{supp} \varphi)}\right) \\
& \leq\|u\|_{L^{p}\left(\mathbb{R}^{3}\right)}\left(\|v\|_{L^{q}\left(\mathbb{R}^{3}\right)}+C_{q, \varphi}\left\|\partial_{i} \Delta^{-1} v\right\|_{L^{q^{*}}(\operatorname{supp} \varphi)}\right) \\
& \leq C\|u\|_{L^{p}\left(\mathbb{R}^{3}\right)}\|v\|_{L^{q}\left(\mathbb{R}^{3}\right)}
\end{aligned}
$$

Corollary 2.6. $\partial_{i}\left[\varphi, \mathbb{P}_{\nabla}\right]$ and $\left[\varphi, \mathbb{P}_{\nabla}\right] \partial_{i}$ are both bounded linear operator from $L^{p}$ to $L^{p}$ for any $1<p<\infty$.

Proof. Id $=\mathbb{P}_{\nabla}+\mathbb{P}_{\text {curl }}$ commutes with $\varphi$, so $\left[\varphi, \mathbb{P}_{\nabla}\right]=-\left[\varphi, \mathbb{P}_{\text {curl }}\right]$.

Because of the smoothing effect of the Laplace potential, we have the following.

Lemma 2.7. Let $\varphi \in C_{c}^{\infty}\left(\mathbb{R}^{3}\right)$ be supported away from some openset $\Omega \subset \mathbb{R}^{3}$, that $i s$, $\operatorname{dist}(\operatorname{supp} \varphi, \Omega)=d>0$. Then for any $f \in L_{\mathrm{loc}}^{1}\left(\mathbb{R}^{3}\right), k>0$,

$$
\left\|\Delta^{-1}(\varphi f)\right\|_{C^{k}(\Omega)} \lesssim_{k, d}\|f\|_{L^{1}(\operatorname{supp} \varphi)} .
$$

We also have

$$
\left\|\mathbb{P}_{\nabla}(\varphi f)\right\|_{C^{k}(\Omega)},\left\|\mathbb{P}_{\text {curl }}(\varphi f)\right\|_{C^{k}(\Omega)} \lesssim_{k, d}\|f\|_{L^{1}(\operatorname{supp} \varphi)} .
$$

\section{Proof of the Main Results}

In this section, we show that the Local Theorem 1.3 leads to the main results. First, we show the pivot quantity is indeed enough to bound $\nabla^{n} \omega$.

Lemma 3.1. There exists $\eta_{2}>0$ such that the following holds. Let $\frac{11}{6}<p<2$, $\frac{2-p}{p-1}<\nu \leq \frac{7 p-12}{6-p}$. If $u$ is a suitable solution to the Navier-Stokes equations in $(-9,0) \times \mathbb{R}^{3}$ satisfying the following conditions,

$$
\begin{aligned}
& \int_{B_{1}} u(t, x) \phi(x) \mathrm{d} x=0, \quad \text { a.e. } t \in(-9,0), \\
& \delta^{-\nu}\left(\int_{Q_{3}}|\nabla u|^{p} \mathrm{~d} x \mathrm{~d} t\right)^{\frac{1}{p}} \leq \eta_{2} \\
& \delta \int_{Q_{3}}|\nabla u|^{2} \mathrm{~d} x \mathrm{~d} t \leq \eta_{2}
\end{aligned}
$$

for some $\delta \leq \eta_{2}$, then we have for any $n \geq 0$,

$$
\left\|\nabla^{n} \omega\right\|_{L_{t, x}^{\infty}\left(Q_{8-n-2}\right)} \leq C_{n} .
$$

Here $C_{n}$ is the same constant in Theorem 1.3 .

Proof. First, we claim that

$$
\delta\|\omega\|_{L^{\infty}\left(-4,0 ; L^{1}\left(B_{2}\right)\right)} \leq C \eta_{2} .
$$


Formally, we can take the dot product of both sides of the vorticity equation (1.1) with $\omega^{0}:=\frac{\omega}{|\omega|}$, and recalling the convexity inequality $\omega^{0} \cdot \Delta \omega \leq \Delta|\omega|$, we have

$$
\left(\partial_{t}+u \cdot \nabla-\Delta\right)|\omega|-\omega \cdot \nabla u \cdot \omega^{0} \leq 0 .
$$

Let $\psi \in C_{c}^{\infty}\left((-9,0] \times \mathbb{R}^{3}\right)$ be a cut-off function such that $\mathbf{1}_{Q_{2}} \leq \psi \leq \mathbf{1}_{Q_{3}}$. Multiply (3.5) by $\psi$ and then integrate in space,

$$
\begin{aligned}
\frac{\mathrm{d}}{\mathrm{d} t} \int \psi|\omega| \mathrm{d} x & \leq \int\left[\left(\partial_{t}+u \cdot \nabla+\Delta\right) \psi\right]|\omega| \mathrm{d} x+\int \psi \omega \cdot \nabla u \cdot \omega^{0} \mathrm{~d} x \\
& \leq C \int_{B_{3}} 1+|u|^{2}+|\nabla u|^{2} \mathrm{~d} x \leq C\left(1+\int_{B_{3}}|\nabla u|^{2} \mathrm{~d} x\right) .
\end{aligned}
$$

for some large universal constant $C>1$. The last step uses Poincaré's inequality and (3.1). Integrate in time we obtain

$$
\|\omega\|_{L^{\infty}\left(-4,0 ; L^{1}\left(B_{2}\right)\right)} \leq C\left(1+\frac{\eta_{2}}{\delta}\right) \leq 2 C \frac{\eta_{2}}{\delta} .
$$

This proves the claim. A more rigorous proof can be obtained by difference quotient same as in Constantin [Con90] or Lions [Lio96] Theorem 3.6, so we omit the details.

Now we interpolate between (3.2) and (3.4). Let $\theta=\frac{1}{1+\nu}$,

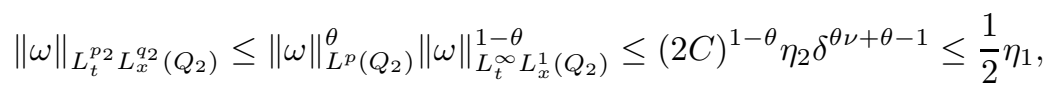

where we choose $\eta_{2}=\frac{\eta_{1}}{4 C+1} \leq \frac{1}{2} \eta_{1}$ from Theorem 1.3, and $p_{2}, q_{2}$ are determined by

$$
\frac{1}{p_{2}}=\frac{\theta}{p}, \quad \frac{1}{q_{2}}=\frac{\theta}{p}+1-\theta .
$$

Combine the above with (3.2) we have

$$
\|\nabla u\|_{L_{t}^{p} L_{x}^{p}\left(Q_{2}\right)}+\|\omega\|_{L_{t}^{p_{2}} L_{x}^{q_{2}}\left(Q_{2}\right)} \leq \frac{1}{2} \eta_{1}+\frac{1}{2} \eta_{1}=\eta_{1} .
$$

By the choice of $\theta$ and the range of $\nu$,

$$
\begin{aligned}
& \frac{1}{p}+\frac{1}{p_{2}}=\frac{1}{p}+\frac{1}{p(1+\nu)}=\frac{2+\nu}{p(1+\nu)}<1, \\
& \frac{1}{p}+\frac{1}{q_{2}}=\frac{1}{p}+\frac{1+\nu p}{p(1+\nu)}=\frac{2+\nu+\nu p}{p(1+\nu)} \leq \frac{7}{6} .
\end{aligned}
$$

One can also easily check that $p<2$ implies $q_{2}<2$, and thus by (3.1) and (3.6) the requirements of the Local Theorem 1.3 are satisfied with $p_{1}=q_{1}=p$, and it completes the proof of the lemma.

Now we transform this lemma into the global coordinate. Recall that $Q_{\varepsilon}(t, x)$ is defined by (2.1).

Corollary 3.2. There exists $\eta_{3}>0$ such that the following holds. If for some $\delta \leq \eta_{2}$,

$$
\delta^{-2 \nu}\left(f_{Q_{\varepsilon}(t, x)}|\nabla u|^{p} \mathrm{~d} x \mathrm{~d} t\right)^{\frac{2}{p}}+\delta f_{Q_{\varepsilon}(t, x)}|\nabla u|^{2} \mathrm{~d} x \mathrm{~d} t \leq \eta_{3} \varepsilon^{-4}
$$

then

$$
\left|\nabla^{n} \omega(t, x)\right| \leq C_{n} \varepsilon^{-n-2} .
$$


Proof. Define $\tilde{u}$ by (1.3). Then (3.7) implies

$$
\begin{gathered}
\delta^{-2 \nu}\left(f_{Q_{3}}|\nabla \tilde{u}|^{p} \mathrm{~d} x \mathrm{~d} t\right)^{\frac{2}{p}} \leq \eta_{3}, \quad \delta f_{Q_{3}}|\nabla \tilde{u}|^{2} \mathrm{~d} x \mathrm{~d} t \leq \eta_{3} \\
\Rightarrow \delta^{-\nu}\left(\int_{Q_{3}}|\nabla \tilde{u}|^{p} \mathrm{~d} x \mathrm{~d} t\right)^{\frac{1}{p}} \leq \eta_{3}^{\frac{1}{2}}\left|Q_{3}\right|^{\frac{1}{p}}, \quad \delta \int_{Q_{3}}|\nabla \tilde{u}|^{2} \mathrm{~d} x \mathrm{~d} t \leq \eta_{3}\left|Q_{3}\right| .
\end{gathered}
$$

Moreover, (3.1) is satisfied by $\tilde{u}$. Therefore, if we choose $\eta_{3}$ such that

$$
\max \left\{\eta_{3}^{\frac{1}{2}}\left|Q_{3}\right|^{\frac{1}{p}}, \eta_{3}\left|Q_{3}\right|\right\}=\eta_{2}
$$

then by Lemma $3.1 \tilde{\omega}:=\operatorname{curl} \tilde{u}$ has bounded derivatives at $(0,0)$, and thus finish the proof of the corollary by scaling.

Then we use the maximal function to go from the local bound to a global bound.

Proof of Theorem 1.1. First, we fix $\frac{11}{6}<p<2, \frac{2-p}{p-1}<\nu \leq \frac{7 p-12}{6-p}$. Let $\eta<<1$ be a small constant to be specified later. Finally we fix a $0<\delta<\infty$. For $(t, x) \in(0, T) \times \mathbb{R}^{3}$, define

$$
I(\varepsilon)=\varepsilon^{4}\left[\delta^{-2 \nu}\left(f_{Q_{\varepsilon}(t, x)}|\mathcal{M}(\nabla u)|^{p}\right)^{\frac{2}{p}}+\delta f_{Q_{\varepsilon}(t, x)}|\mathcal{M}(\nabla u)|^{2}\right] .
$$

If $(t, x)$ is both a $\mathcal{Q}$-Lebesgue point of $|\mathcal{M}(\nabla u)|^{p}$ and of $|\mathcal{M}(\nabla u)|^{2}$, then we claim that there exists a positive $\varepsilon=\varepsilon_{(t, x)}$ such that one of the two cases is true:

Case 1. $3 \varepsilon_{(t, x)}<t^{\frac{1}{2}}$, and $I\left(\varepsilon_{(t, x)}\right)=\eta$.

Case 2. $3 \varepsilon_{(t, x)}=t^{\frac{1}{2}}$, and $I\left(\varepsilon_{(t, x)}\right) \leq \eta$.

This is because by Theorem 2.2

$$
\lim _{\varepsilon \rightarrow 0} I(\varepsilon)=0^{4}\left[\delta^{-2 \nu}\left(|\mathcal{M}(|\nabla u|)(t, x)|^{p}\right)^{\frac{2}{p}}+\delta|\mathcal{M}(|\nabla u|)(t, x)|^{2}\right]=0,
$$

and $I(\varepsilon)$ is a continuous function of $\varepsilon$.

One the one hand, in both cases we have $I(\varepsilon) \leq \eta$, which implies that

$$
\delta^{-\nu} \varepsilon^{2}\left(f_{Q_{\varepsilon}(t, x)}|\mathcal{M}(\nabla u)|^{p}\right)^{\frac{1}{p}} \leq \sqrt{\eta}, \quad \delta^{\frac{1}{2}} \varepsilon^{2}\left(f_{Q_{\varepsilon}(t, x)}|\mathcal{M}(\nabla u)|^{2}\right)^{\frac{1}{2}} \leq \sqrt{\eta} .
$$

If we set $\eta<\eta_{0}^{2}$, then depending on $\delta \geq 1$ or $\delta \leq 1$, one of the two would imply admissibility condition (2.2) by Jensen's inequality. Therefore $Q_{\varepsilon}(t, x)$ is admissible and

$$
I(\varepsilon) \leq \varepsilon^{4}\left[\delta^{-2 \nu} \mathcal{M}_{\mathcal{Q}}\left(\mathcal{M}(\nabla u)^{p}\right)^{\frac{2}{p}}+\delta \mathcal{M}_{\mathcal{Q}}\left(\mathcal{M}(\nabla u)^{2}\right)\right],
$$

so we can combine two canses and conclude

$$
\varepsilon_{(t, x)}^{-4} \leq \max \left\{\frac{1}{\eta}\left[\delta^{-2 \nu} \mathcal{M}_{\mathcal{Q}}\left(\mathcal{M}(\nabla u)^{p}\right)^{\frac{2}{p}}+\delta \mathcal{M}_{\mathcal{Q}}\left(\mathcal{M}(\nabla u)^{2}\right)\right], 81 t^{-2}\right\} .
$$

On the other hand, if we set $\eta<\eta_{3}$, then in both cases $I(\varepsilon) \leq \eta_{3}$. If $\delta \leq \eta_{2}$ one would have

$$
\left|\nabla^{n} \omega(t, x)\right| \leq C_{n} \varepsilon^{-n-2}
$$


by Corollary 3.2. If $\delta>\eta_{2}$, notice that by Jensen's inequality,

so

$$
\left(f_{Q_{\varepsilon}(t, x)}|\mathcal{M}(\nabla u)|^{p}\right)^{\frac{2}{p}} \leq f_{Q_{\varepsilon}(t, x)}|\mathcal{M}(\nabla u)|^{2},
$$

$$
\begin{aligned}
I(\varepsilon) & \geq \varepsilon^{4}\left[\left(\delta^{-2 \nu}+\delta-\eta_{2}\right)\left(f_{Q_{\varepsilon}(t, x)}|\mathcal{M}(\nabla u)|^{p}\right)^{\frac{2}{p}}+\eta_{2} f_{Q_{\varepsilon}(t, x)}|\mathcal{M}(\nabla u)|^{2}\right] \\
& \geq \varepsilon^{4}\left[\left(1-\eta_{2}\right)\left(f_{Q_{\varepsilon}(t, x)}|\mathcal{M}(\nabla u)|^{p}\right)^{\frac{2}{p}}+\eta_{2} f_{Q_{\varepsilon}(t, x)}|\mathcal{M}(\nabla u)|^{2}\right] \\
& \geq\left(1-\eta_{2}\right) \eta_{2}^{2 \nu} \varepsilon^{4}\left[\eta_{2}^{-2 \nu}\left(f_{Q_{\varepsilon}(t, x)}|\mathcal{M}(\nabla u)|^{p}\right)^{\frac{2}{p}}+\eta_{2} f_{Q_{\varepsilon}(t, x)}|\mathcal{M}(\nabla u)|^{2}\right] .
\end{aligned}
$$

If we require $\eta<\left(1-\eta_{2}\right) \eta_{2}^{2 \nu} \eta_{3}$, then

$$
\varepsilon^{4}\left[\eta_{2}^{-2 \nu}\left(f_{Q_{\varepsilon}(t, x)}|\mathcal{M}(\nabla u)|^{p}\right)^{\frac{2}{p}}+\eta_{2} f_{Q_{\varepsilon}(t, x)}|\mathcal{M}(\nabla u)|^{2}\right] \leq \eta_{3} .
$$

again by Corollary 3.2 we would still have (3.9). In conclusion, we choose

$$
\eta=\min \left\{\eta_{0}^{2},\left(1-\eta_{2}\right) \eta_{2}^{2 \nu} \eta_{3}\right\},
$$

then for any $0<\delta<\infty$ one would have

$$
\left|\nabla^{n} \omega(t, x)\right|^{\frac{4}{n+2}} \leq C_{n}^{\frac{4}{n+2}} \max \left\{\frac{1}{\eta}\left[\delta^{-2 \nu} \mathcal{M}_{\mathcal{Q}}\left(\mathcal{M}(\nabla u)^{p}\right)^{\frac{2}{p}}+\delta \mathcal{M}_{\mathcal{Q}}\left(\mathcal{M}(\nabla u)^{2}\right)\right], 81 t^{-2}\right\}
$$

by putting (3.9) and (3.8) together. Denote $f=\left|\nabla^{n} \omega\right|^{\frac{4}{n+2}}$, and we denote $f_{1}=$ $\mathcal{M}_{\mathcal{Q}}\left(\mathcal{M}(|\nabla u|)^{p}\right)^{\frac{2}{p}}, f_{2}=\mathcal{M}_{\mathcal{Q}}\left(\mathcal{M}(|\nabla u|)^{2}\right)$. Then we have almost everywhere

$$
f \mathbf{1}_{\left\{f>C_{n} t^{-2}\right\}} \lesssim_{n} \delta^{-2 \nu} f_{1}+\delta f_{2} .
$$

By Theorem 2.1.

$$
\begin{aligned}
\left\|f_{1}\right\|_{L^{1}} & \leq C_{p}\left\|\mathcal{M}(\nabla u)^{p}\right\|_{L^{\frac{2}{p}}}^{\frac{2}{p}} \lesssim C_{p}\left\|\mathcal{M}(\nabla u)^{2}\right\|_{L^{1}} \leq C_{p}\|\nabla u\|_{L^{2}}^{2}, \\
\left\|f_{2}\right\|_{L^{1, \infty}} & \leq C_{1}\left\|\mathcal{M}(\nabla u)^{2}\right\|_{L^{1}} \leq C_{1}\|\nabla u\|_{L^{2}}^{2} .
\end{aligned}
$$

Finally, by the interpolation between Lorentz spaces Lemma 2.3 ,

$$
\left\|f \mathbf{1}_{\left\{f>C_{n} t^{-2}\right\}}\right\|_{L^{1,1+2 \nu}} \lesssim_{p, n}\|\nabla u\|_{L^{2}\left((0, T) \times \mathbb{R}^{3}\right)}^{2} \leq\left\|u_{0}\right\|_{L^{2}\left(\mathbb{R}^{3}\right)}^{2} .
$$

This proves the theorem for $q \geq 1+2 \nu$. Recall that $p$ can be arbitrarily chosen between $\frac{11}{6}$ and 2 , and $\nu$ can be chosen between $\frac{2-p}{p-1}$ and $\frac{7 p-12}{6-p}$, so $\nu$ can be arbitrarily small, therefore we prove the theorem for any $q>1$.

Estimates on $\nabla^{2} u$ can be obtained by a Riesz transform of $\Delta u=-\operatorname{curl} \omega$.

Proof of Corollary 1.2. We can put $K \subset\left(t_{0}, T\right) \times B_{R}$ for some $t_{0}, T, R>0$. Denote $Q=\left(t_{0}, T\right) \times B_{2 R}$. Let $\rho \in C_{c}^{\infty}\left(\mathbb{R}^{3}\right)$ be a smooth spatial cut-off function between $\mathbf{1}_{B_{R}} \leq \rho \leq \mathbf{1}_{B_{2 R}}$. Then

$$
\|\Delta(\rho u)\|_{L^{\frac{4}{3}, q}\left(\left(t_{0}, T\right) \times \mathbb{R}^{3}\right)} \lesssim \rho\|\Delta u\|_{L^{\frac{4}{3}, q}(Q)}+\|\nabla u\|_{L^{\frac{4}{3}, q}(Q)}+\|u\|_{L^{\frac{4}{3}, q}(Q)} .
$$


Since $\Delta u=-\operatorname{curl} \omega$, the case $n=1$ of Theorem 1.1 gives

SO

$$
\left\|\Delta u \mathbf{1}_{\left\{|\Delta u|>C_{1} t^{-\frac{3}{2}}\right\}}\right\|_{L^{\frac{4}{3}, q}\left((0, T) \times \mathbb{R}^{3}\right)} \leq C_{q}\left\|u_{0}\right\|_{L^{2}\left(\mathbb{R}^{3}\right)}^{\frac{3}{2}},
$$

$$
\|\Delta u\|_{L^{\frac{4}{3}, q}(Q)} \leq C_{q}\left\|u_{0}\right\|_{L^{2}\left(\mathbb{R}^{3}\right)}^{\frac{3}{2}}+C_{1}\left\|t^{-\frac{3}{2}}\right\|_{L^{\frac{4}{3}(Q)}} \lesssim C_{q}\left\|u_{0}\right\|_{L^{2}\left(\mathbb{R}^{3}\right)}^{\frac{3}{2}}+C_{1}\left(\frac{R^{3}}{t_{0}}\right)^{\frac{3}{4}} .
$$

As for lower order terms,

$$
\begin{aligned}
\|\nabla u\|_{L^{\frac{4}{3}}(Q)} & \lesssim\|\nabla u\|_{L^{2}(Q)}, \\
\|u\|_{L^{\frac{4}{3}}(Q)} & \leq\|u\|_{L_{t}^{\infty} L_{x}^{2}(Q)} .
\end{aligned}
$$

For Leray-Hopf solution, $\|\nabla u\|_{L_{t}^{\infty} L_{x}^{2} \cap L_{t}^{2} \dot{H}_{x}^{1}\left((0, T) \times \mathbb{R}^{3}\right)} \leq\left\|u_{0}\right\|_{L^{2}}$, so

$$
\|\Delta(\rho u)\|_{L^{\frac{4}{3}, q}\left(\left(t_{0}, T\right) \times \mathbb{R}^{3}\right)} \lesssim_{q, K}\left\|u_{0}\right\|_{L^{2}\left(\mathbb{R}^{3}\right)}^{\frac{3}{2}}+1+\left\|u_{0}\right\|_{L^{2}\left(\mathbb{R}^{3}\right)} \lesssim\left\|u_{0}\right\|_{L^{2}\left(\mathbb{R}^{3}\right)}^{\frac{3}{2}}+1 .
$$

Because Riesz transform is bounded from $L^{\frac{4}{3}, q}\left(\left(t_{0}, T\right) \times \mathbb{R}^{3}\right)$ to itself by Lemma 2.4

$$
\left\|\nabla^{2} u\right\|_{L^{\frac{4}{3}, q}(K)} \leq\left\|\nabla^{2}(\rho u)\right\|_{L^{\frac{4}{3}, q}(Q)} \lesssim_{q, K}\left\|u_{0}\right\|_{L^{2}\left(\mathbb{R}^{3}\right)}^{\frac{3}{2}}+1 .
$$

Remark 3.3. For smooth solutions to the Navier-Stokes equation, we have $L^{1, q}$ estimate for the third derivatives for any $q>1$,

$$
\left\|\nabla^{2} \omega \mathbf{1}_{\left\{\left|\nabla^{2} \omega\right|>C t^{-2}\right\}}\right\|_{L^{1, q}\left((0, T) \times \mathbb{R}^{3}\right)} \leq C_{q}\left\|u_{0}\right\|_{L^{2}}^{2} .
$$

\section{Local Study: Part One, Initial Energy}

The following three sections are dedicated to the proof of the Local Theorem 1.3. In Vas10, the proof of the local theorem consists of the following three parts: Step 1 . Show the velocity $u$ is locally small in the energy space $\mathcal{E}=L_{t}^{\infty} L_{x}^{2} \cap L_{t}^{2} H_{x}^{1}$. Step 2. Use De Giorgi iteration and the truncation method developed in Vas07. to show $u$ is locally bounded in $L^{\infty}$.

Step 3. Bootstrap to higher regularity by differentiating the original equation.

In our case, directly working with $u$ is difficult due to the lack of control on the pressure, which is nonlocal. Therefore, we would like to work on vorticity, whose evolution is governed by (1.6) and only involves local quantities. Since $\omega$ is one derivative of $u$, we have less integrability to do any parabolic regularization, and we don't have the local energy inequality to perform De Giorgi iteration. This motivates us to work on minus one derivative of $\omega$, but instead of $\omega$ we use a localization of $\omega$. Similar as CLRM18, we introduce a new local quantity

$$
v:=-\operatorname{curl} \varphi^{\sharp} \Delta^{-1} \varphi \operatorname{curl} u=-\operatorname{curl} \varphi^{\sharp} \Delta^{-1} \varphi \omega .
$$

where $\varphi$ and $\varphi^{\sharp}$ are a pair of fixed smooth spatial cut-off functions, which are defined between $\mathbf{1}_{B_{\frac{6}{5}}} \leq \varphi \leq \mathbf{1}_{B_{\frac{5}{4}}}, \mathbf{1}_{B_{\frac{4}{3}}} \leq \varphi^{\sharp} \leq \mathbf{1}_{B_{\frac{3}{2}}}$. This $v$ is divergence free and compactly supported. It will help us get rid of the pressure $P$, while staying in the same space as $u$ : it scales the same as $u$, has the same regularity, inherit a local energy inequality from $u$, and its evolution only depends on local information. We will follow the same three steps above, but we will work on $v$ instead of $u$. 
For convenience, from now on we will use $\eta$ to denote a small universal constant depending only on the smallness of $\eta_{1}$, such that $\lim _{\eta_{1} \rightarrow 0} \eta=0$. Similar as the constant $C$, the value of $\eta$ may change from line to line. The purpose of this section is to obtain the smallness of $v$ in the energy space $\mathcal{E}$, which is the following proposition.

Proposition 4.1. Under the same assumptions of the Local Theorem 1.3, we have

$$
\|v\|_{\mathcal{E}\left(Q_{1}\right)}^{2}=\sup _{t \in(-1,0)} \int_{B_{1}}|v(t)|^{2} \mathrm{~d} x+\int_{Q_{1}}|\nabla v|^{2} \mathrm{~d} x \leq \eta .
$$

For convenience, define $q_{3}, q_{4}, q_{5}$ by

$$
\frac{1}{q_{3}}=\frac{1}{q_{1}}-\frac{1}{3}, \quad \frac{1}{q_{4}}=\frac{1}{q_{2}}-\frac{1}{3}, \quad \frac{1}{q_{5}}=\left(\frac{1}{q_{3}}-\frac{1}{3}\right)_{+} .
$$

4.1. Equations of $\mathbf{v}$. We use (2.3) in (1.1) to rewrite the equation of $u$, then take the curl to rewrite the equation of $\omega$, finally apply $-\operatorname{curl} \varphi^{\sharp} \Delta^{-1} \varphi$ on the vorticity equation to obtain the equation of $v$.

$$
\begin{aligned}
\partial_{t} u+\mathbb{P}_{\text {curl }}(\omega \times u) & =\Delta u, \\
\partial_{t} \omega+\operatorname{curl}(\omega \times u) & =\Delta \omega, \\
\partial_{t} v-\operatorname{curl} \varphi^{\sharp} \Delta^{-1} \varphi \operatorname{curl}(\omega \times u) & =-\operatorname{curl} \varphi^{\sharp} \Delta^{-1} \varphi \Delta \omega .
\end{aligned}
$$

The second term of (4.2) is

$$
\operatorname{curl} \varphi^{\sharp} \Delta^{-1} \varphi \operatorname{curl}(\omega \times u)=\mathbf{B}-\mathbb{P}_{\text {curl }}(\varphi \omega \times u)
$$

where $\mathbf{B}$ denotes the quadratic commutator

$$
\begin{aligned}
\mathbf{B} & :=-\operatorname{curl}\left(1-\varphi^{\sharp}\right) \Delta^{-1} \varphi \operatorname{curl}(\omega \times u)+\operatorname{curl} \Delta^{-1}[\varphi, \operatorname{curl}](\omega \times u) \\
& =-\operatorname{curl}\left(1-\varphi^{\sharp}\right) \Delta^{-1} \varphi \operatorname{curl}(\omega \times u)+\operatorname{curl} \Delta^{-1}(-\nabla \varphi \times(\omega \times u))
\end{aligned}
$$

Here we used (2.5). The right hand side of (4.2) is

$$
-\operatorname{curl} \varphi^{\sharp} \Delta^{-1} \varphi \Delta \omega=\Delta v+\mathbf{L}
$$

where $\mathbf{L}$ denotes the linear commutator

$$
\begin{aligned}
\mathbf{L} & :=\left[-\operatorname{curl} \varphi^{\sharp} \Delta^{-1} \varphi, \Delta\right] \omega \\
& =-\operatorname{curl}\left[\varphi^{\sharp} \Delta^{-1} \varphi, \Delta\right] \omega \\
& =-\operatorname{curl}\left[\varphi^{\sharp}, \Delta\right] \Delta^{-1} \varphi \omega-\operatorname{curl} \varphi^{\sharp} \Delta^{-1}[\varphi, \Delta] \omega \\
& =-\operatorname{curl}\left[\varphi^{\sharp}, \Delta\right] \Delta^{-1} \varphi \omega+\operatorname{curl} \varphi^{\sharp} \Delta^{-1}(2 \operatorname{div}(\nabla \varphi \otimes \omega)-(\Delta \varphi) \omega) .
\end{aligned}
$$

Here we used (2.6). Therefore we have the equation for $v$ as the following,

$$
\partial_{t} v+\mathbb{P}_{\text {curl }}(\varphi \omega \times u)=\mathbf{B}+\mathbf{L}+\Delta v .
$$

We observe the following localization decomposition.

Lemma 4.2. We can decompose

$$
\varphi u=v+w, \quad \varphi \omega=\operatorname{curl} v+\varpi,
$$

where $w$ and $\varpi$ are harmonic inside $B_{1}$. 
Proof. We can compute $v$ by

$$
\begin{aligned}
v & =-\operatorname{curl} \varphi^{\sharp} \Delta^{-1} \varphi \operatorname{curl} u \\
& =\operatorname{curl}\left(1-\varphi^{\sharp}\right) \Delta^{-1} \varphi \operatorname{curl} u-\operatorname{curl} \Delta^{-1} \varphi \operatorname{curl} u \\
& =\operatorname{curl}\left(1-\varphi^{\sharp}\right) \Delta^{-1} \varphi \omega-\operatorname{curl} \Delta^{-1}[\varphi, \operatorname{curl}] u+\mathbb{P}_{\text {curl }}(\varphi u) \\
& =\operatorname{curl}\left(1-\varphi^{\sharp}\right) \Delta^{-1} \varphi \omega+\operatorname{curl} \Delta^{-1}(\nabla \varphi \times u)-\mathbb{P}_{\nabla}(\varphi u)+\varphi u \\
& =\operatorname{curl}\left(1-\varphi^{\sharp}\right) \Delta^{-1} \varphi \omega+\operatorname{curl} \Delta^{-1}(\nabla \varphi \times u)-\nabla \Delta^{-1}(\nabla \varphi \cdot u)+\varphi u
\end{aligned}
$$

using $\operatorname{div} u=0$. We denote

$$
w:=-\operatorname{curl}\left(1-\varphi^{\sharp}\right) \Delta^{-1} \varphi \omega-\operatorname{curl} \Delta^{-1}(\nabla \varphi \times u)+\nabla \Delta^{-1}(\nabla \varphi \cdot u),
$$

which implies the first decomposition $\varphi u=v+w$. By taking the curl,

$$
\begin{aligned}
\operatorname{curl}(\varphi u) & =\operatorname{curl} v+\operatorname{curl} w, \\
\nabla \varphi \times u+\varphi \omega & =\operatorname{curl} v-\operatorname{curl} \operatorname{curl}\left(1-\varphi^{\sharp}\right) \Delta^{-1} \varphi \omega-\operatorname{curl} \operatorname{curl} \Delta^{-1}(\nabla \varphi \times u) \\
& =\operatorname{curl} v-\operatorname{curl} \operatorname{curl}\left(1-\varphi^{\sharp}\right) \Delta^{-1} \varphi \omega+\mathbb{P}_{\operatorname{curl}}(\nabla \varphi \times u) .
\end{aligned}
$$

We denote

$$
\begin{aligned}
\varpi & :=-\operatorname{curl} \operatorname{curl}\left(1-\varphi^{\sharp}\right) \Delta^{-1} \varphi \omega-\mathbb{P}_{\nabla}(\nabla \varphi \times u) \\
& =-\operatorname{curl} \operatorname{curl}\left(1-\varphi^{\sharp}\right) \Delta^{-1} \varphi \omega-\nabla \Delta^{-1} \operatorname{div}(\nabla \varphi \times u) \\
& =-\operatorname{curl} \operatorname{curl}\left(1-\varphi^{\sharp}\right) \Delta^{-1} \varphi \omega+\nabla \Delta^{-1}(\nabla \varphi \cdot \omega)
\end{aligned}
$$

which implies the second decomposition $\varphi \omega=\operatorname{curl} v+\varpi$. We can easily see that $\Delta w$ and $\Delta \varpi$ are both the sum of a smooth function supported outside $B_{\frac{3}{2}}$ and the Newtonian potential of something supported inside $\operatorname{supp}(\nabla \varphi) \subset B_{\frac{5}{4}} \backslash B_{\frac{6}{5}}$, so they are harmonic inside $B_{1}$.

Using this decomposition, we can continue to expand

$$
\begin{aligned}
\mathbb{P}_{\text {curl }}(\varphi \omega \times u) & =\varphi \omega \times u-\mathbb{P}_{\nabla}(\varphi \omega \times u) \\
& =\omega \times v+\omega \times w-\frac{1}{2} \mathbb{P}_{\nabla}((\operatorname{curl} v+\varpi) \times u+\omega \times(v+w)) \\
& =\omega \times v-\frac{1}{2} \mathbb{P}_{\nabla}(\operatorname{curl} v \times u+\omega \times v)-\mathbf{W},
\end{aligned}
$$

where $\mathbf{W}$ denotes the remainders involving $w$ and $\varpi$,

$$
\mathbf{W}:=-\omega \times w+\frac{1}{2} \mathbb{P}_{\nabla}(\varpi \times u+\omega \times w) .
$$

By subtracting (2.4) from (2.3), for divergence free $u, v$ we have

$$
\operatorname{curl} v \times u+\operatorname{curl} u \times v=-\nabla(u \cdot v)+2 u \cdot \nabla v+\operatorname{curl}(u \times v),
$$

so

$$
\begin{aligned}
\mathbb{P}_{\text {curl }}(\varphi \omega \times u) & =\omega \times v+\frac{1}{2} \nabla(u \cdot v)-\mathbb{P}_{\nabla} \operatorname{div}(u \otimes v)-\mathbf{W} \\
& =\omega \times v+\nabla\left(\frac{1}{2} u \cdot v-\Delta^{-1} \operatorname{div} \operatorname{div}(u \otimes v)\right)-\mathbf{W} .
\end{aligned}
$$

For convenience, denote the Riesz operator

$$
\mathbf{R}=\frac{1}{2} \operatorname{tr}-\Delta^{-1} \operatorname{div} \operatorname{div}
$$


Finally, we have the equation of $v$ as

$$
\partial_{t} v+\omega \times v+\nabla \mathbf{R}(u \otimes v)=\mathbf{B}+\mathbf{L}+\mathbf{W}+\Delta v, \quad \operatorname{div} v=0 .
$$

We now check the spatial integrability of these new terms.

Lemma 4.3. For any $1<p<\infty$,

$$
\begin{aligned}
& \|v\|_{L^{p}},\|\nabla w\|_{L^{p}},\|\varpi\|_{L^{p}} \lesssim\|\omega\|_{L^{1}\left(B_{2}\right)}+\|u\|_{L^{p}\left(B_{2}\right)}, \\
& \|\nabla v\|_{L^{p}},\|\nabla \varpi\|_{L^{p}} \lesssim\|\omega\|_{L^{p}\left(B_{2}\right)}, \\
& \left\|\nabla^{2} w\right\|_{L^{p}} \lesssim\|u\|_{W^{1, p}\left(B_{2}\right) .}
\end{aligned}
$$

If we denote $q=\min \left\{1, \frac{1}{p}+\frac{1}{3}\right\}_{+}^{-1}$, then

$$
\begin{aligned}
\|\mathbf{B}\|_{L^{p}\left(B_{2}\right)} & \lesssim\|\omega \times u\|_{L^{q}\left(B_{2}\right)}, \\
\|\mathbf{L}\|_{L^{p}\left(B_{2}\right)} & \lesssim\|\omega\|_{L^{p}\left(B_{2}\right)}, \\
\|\mathbf{W}\|_{L^{p}\left(B_{2}\right)} & \lesssim\|\omega \times w\|_{L^{p}\left(B_{2}\right)}+\|\varpi \times u\|_{L^{p}\left(B_{2}\right)} .
\end{aligned}
$$

Proof. $v, w, \varpi$ are all supported inside $B_{2}$, so

$$
\begin{aligned}
\|v\|_{L^{p}} \leq & \|\varphi u\|_{L^{p}}+\|w\|_{L^{p}} \lesssim\|u\|_{L^{p}\left(B_{2}\right)}+\|\nabla w\|_{L^{p}}, \\
\|\nabla w\|_{L^{p}} \leq & \left\|\nabla \operatorname{curl}\left(1-\varphi^{\sharp}\right) \Delta^{-1} \varphi \omega\right\|_{L^{p}\left(B_{2}\right)}+\left\|\nabla \operatorname{curl} \Delta^{-1}(\nabla \varphi \times u)\right\|_{L^{p}} \\
& \quad+\left\|\nabla^{2} \Delta^{-1}(\nabla \varphi \cdot u)\right\|_{L^{p}} \\
\lesssim & \left\|\left(1-\varphi^{\sharp}\right) \Delta^{-1} \varphi \omega\right\|_{C^{2}}+\|\nabla \varphi \times u\|_{L^{p}}+\|\nabla \varphi \cdot u\|_{L^{p}} \\
\lesssim & \|\omega\|_{L^{1}\left(B_{2}\right)}+\|u\|_{L^{p}\left(B_{2}\right)}, \\
\|\varpi\|_{L^{p}} \leq\left\|\operatorname{curl} \operatorname{curl}\left(1-\varphi^{\sharp}\right) \Delta^{-1} \varphi \omega\right\|_{L^{p}\left(B_{2}\right)}+\left\|\nabla \Delta^{-1}(\nabla \varphi \cdot \omega)\right\|_{L^{p}} & \|\|\left(1-\varphi^{\sharp}\right) \Delta^{-1} \varphi \omega\left\|_{C^{2}}+\right\| \nabla \Delta^{-1} \operatorname{div}(\nabla \varphi \times u) \|_{L^{p}} \\
\leq & \|\omega\|_{L^{1}\left(B_{2}\right)}+\|\nabla \varphi \times u\|_{L^{p}} \\
\leq & \|\omega\|_{L^{1}\left(B_{2}\right)}+\|u\|_{L^{p}\left(B_{2}\right)} .
\end{aligned}
$$

Here we used Lemma 2.7 since $\varphi$ and $1-\varphi^{\sharp}$ are supported away from each other, and we also used the boundedness of Riesz transform by Lemma 2.4. Their derivatives are bounded by

$$
\begin{aligned}
\|\nabla v\|_{L^{p}} & =\left\|\nabla \operatorname{curl} \varphi^{\sharp} \Delta^{-1} \varphi \omega\right\|_{L^{p}} \\
\leq & \left\|\nabla \operatorname{curl} \Delta^{-1} \varphi \omega\right\|_{L^{p}}+\left\|\nabla \operatorname{curl}\left(1-\varphi^{\sharp}\right) \Delta^{-1} \varphi \omega\right\|_{L^{p}\left(B_{2}\right)} \\
\lesssim & \|\omega\|_{L^{p}\left(B_{2}\right)}+\|\omega\|_{L^{1}\left(B_{2}\right)} \lesssim\|\omega\|_{L^{p}\left(B_{2}\right)}, \\
\left\|\nabla^{2} w\right\|_{L^{p}} \leq & \left\|\nabla^{2} \operatorname{curl}\left(1-\varphi^{\sharp}\right) \Delta^{-1} \varphi \omega\right\|_{L^{p}\left(B_{2}\right)}+\left\|\nabla^{2} \operatorname{curl} \Delta^{-1}(\nabla \varphi \times u)\right\|_{L^{p}} \\
& \quad+\left\|\nabla^{3} \Delta^{-1}(\nabla \varphi \cdot u)\right\|_{L^{p}} \\
& \lesssim\|\omega\|_{L^{1}\left(B_{2}\right)}+\|u\|_{W^{1, p}\left(B_{2}\right)} \lesssim\|u\|_{W^{1, p}\left(B_{2}\right)}, \\
\|\nabla \varpi\|_{L^{p}} \leq\left\|\nabla \operatorname{curl} \operatorname{curl}\left(1-\varphi^{\sharp}\right) \Delta^{-1} \varphi \omega\right\|_{L^{p}\left(B_{2}\right)}+\left\|\nabla^{2} \Delta^{-1}(\nabla \varphi \cdot \omega)\right\|_{L^{p}} & \leq\|\omega\|_{L^{1}\left(B_{2}\right)}+\|\omega\|_{L^{p}\left(B_{2}\right)} \lesssim\|\omega\|_{L^{p}\left(B_{2}\right)} .
\end{aligned}
$$

The proof for $\mathbf{B}, \mathbf{L}, \mathbf{W}$ are similar so we omit here. 
Since $u \in \mathcal{E}$, it can be seen from the above lemma that $v, \nabla w, \varpi \in \mathcal{E}$, thus

$$
\begin{aligned}
\|\mathbf{B}\|_{L^{\frac{3}{2}}\left(B_{2}\right)} & \lesssim\|\omega \times u\|_{L^{1}\left(B_{2}\right)} \in L_{t}^{2}, \\
\|\mathbf{L}\|_{L^{2}\left(B_{2}\right)} & \lesssim\|\omega\|_{L^{2}\left(B_{2}\right)} \in L_{t}^{2}, \\
\|\mathbf{W}\|_{L^{\frac{3}{2}}\left(B_{2}\right)} & \lesssim\|\omega \times w\|_{L^{\frac{3}{2}\left(B_{2}\right)}}+\|\varpi \times u\|_{L^{\frac{3}{2}\left(B_{2}\right)}} \in L_{t}^{2},
\end{aligned}
$$

therefore $\mathbf{B}, \mathbf{L}, \mathbf{W} \in L_{t}^{2} L_{\text {loc }, x}^{\frac{3}{2}}$. In the appendix we prove the suitability for $v$ : it satisfies the following local energy inequality,

$$
\partial_{t} \frac{|v|^{2}}{2}+|\nabla v|^{2}+\operatorname{div}[v \mathbf{R}(u \otimes v)] \leq \Delta \frac{|v|^{2}}{2}+v \cdot(\mathbf{B}+\mathbf{L}+\mathbf{W}) .
$$

4.2. Energy Estimate. Multiply (4.5) by $\varphi^{4}$ then integrate over $\mathbb{R}^{3}$ yields

$$
\begin{aligned}
& \frac{\mathrm{d}}{\mathrm{d} t} \int \varphi^{4} \frac{|v|^{2}}{2} \mathrm{~d} x+\int \varphi^{4}|\nabla v|^{2} \mathrm{~d} x \\
& \leq \int \frac{|v|^{2}}{2} \Delta \varphi^{4} \mathrm{~d} x+\int\left(v \cdot \nabla \varphi^{4}\right) \mathbf{R}(u \otimes v) \mathrm{d} x \\
& \quad+\int \varphi^{4} v \cdot \mathbf{B} \mathrm{d} x+\int \varphi^{4} v \cdot \mathbf{L} \mathrm{d} x+\int \varphi^{4} v \cdot \mathbf{W} \mathrm{d} x
\end{aligned}
$$

Let us discuss these terms. For the first four terms on the right hand side,

$$
\begin{aligned}
I_{\Delta} & :=\int \frac{|v|^{2}}{2} \Delta \varphi^{4} \mathrm{~d} x \leq C\left\|\varphi^{2} v\right\|_{L^{2}}\|v\|_{L^{2}}, \\
I_{\mathbf{R}} & :=\int\left(v \cdot \nabla \varphi^{4}\right) \mathbf{R}(u \otimes v) \mathrm{d} x \leq C\left\|\varphi^{2} v\right\|_{L^{2}}\|\mathbf{R}(u \otimes v)\|_{L^{2}} \\
& \leq C\left\|\varphi^{2} v\right\|_{L^{2}}\|u \otimes v\|_{L^{2}}, \\
I_{\mathbf{B}} & :=\int \varphi^{4} v \cdot \mathbf{B} \mathrm{d} x \leq\left\|\varphi^{2} v\right\|_{L^{2}}\left\|\varphi^{2} \mathbf{B}\right\|_{L^{2}} \\
& \leq C\left\|\varphi^{2} v\right\|_{L^{2}}\|\omega \times u\|_{L^{\frac{6}{5}}\left(B_{2}\right)}, \\
I_{\mathbf{L}} & :=\int \varphi^{4} v \cdot \mathbf{L} \mathrm{d} x \leq\left\|\varphi^{\frac{2}{3}}|v|^{\frac{1}{3}}\right\|_{L^{6}}\left\|\left.v\right|^{\frac{2}{3}}\right\|_{L^{q_{3}}}\left\|\varphi^{2} \mathbf{L}\right\|_{L^{q_{2}}} \\
& \leq\left\|\varphi^{2} v\right\|_{L^{2}}^{\frac{1}{3}}\|v\|_{L^{q 3}}^{\frac{2}{3}}\|\omega\|_{L^{q_{2}}\left(B_{2}\right)} .
\end{aligned}
$$

Here we use Hölder's inequality, $\varphi$ is compactly supported in $B_{2}$ and $\frac{1}{q_{2}}+\frac{1}{q_{3}}+\frac{1}{6} \leq 1$. For the $\mathbf{W}$ term,

$$
\begin{aligned}
I_{\mathbf{W}} & :=\int \varphi^{4} v \cdot \mathbf{W} \mathrm{d} x \\
& =-\int \varphi^{4} v \cdot \omega \times w \mathrm{~d} x+\frac{1}{2} \int \varphi^{4} v \cdot \mathbb{P}_{\nabla}(\varpi \times u+\omega \times w) \mathrm{d} x \\
& =-I_{\mathbf{W} 1}+\frac{1}{2} I_{\mathbf{W} 2} .
\end{aligned}
$$

For the first one, we break it as

$$
I_{\mathbf{W} 1}=\int \varphi^{4} v \cdot \omega \times w \mathrm{~d} x=\int \varphi^{3} v \times \operatorname{curl} v \cdot w \mathrm{~d} x+\int \varphi^{3} v \cdot \varpi \times w \mathrm{~d} x .
$$


Using (2.3),

$$
v \times \operatorname{curl} v=\frac{1}{2} \nabla|v|^{2}-(v \cdot \nabla) v
$$

we have

$$
\begin{aligned}
\int \varphi^{3} v \times \operatorname{curl} v \cdot w \mathrm{~d} x & =-\frac{1}{2} \int|v|^{2} \operatorname{div}\left(\varphi^{3} w\right) \mathrm{d} x+\int v \cdot \nabla\left(\varphi^{3} w\right) \cdot v \mathrm{~d} x \\
& \leq C\left\|\varphi^{2} v\right\|_{L^{2}}\left(\|\nabla w \otimes v\|_{L^{2}}+\|w \otimes v\|_{L^{2}}\right) .
\end{aligned}
$$

The remaining is of lower order,

$$
\int \varphi^{3} v \cdot \varpi \times w \mathrm{~d} x \leq C\left\|\varphi^{2} v\right\|_{L^{2}}\|\varpi \times w\|_{L^{2}}
$$

For the second one,

$$
\begin{aligned}
I_{\mathbf{W} 2} & =\int \mathbb{P}_{\nabla}\left(\varphi^{4} v\right) \cdot(\varpi \times u+\omega \times w) \mathrm{d} x \\
& \leq\left\|\mathbb{P}_{\nabla}\left(\varphi^{4} v\right)\right\|_{L^{6}}\|\varpi \times u+\omega \times w\|_{L^{\frac{6}{5}}}
\end{aligned}
$$

where

$$
\left\|\mathbb{P}_{\nabla}\left(\varphi^{4} v\right)\right\|_{L^{6}}=\left\|\left[\mathbb{P}_{\nabla}, \varphi^{2}\right] \varphi^{2} v\right\|_{L^{6}} \lesssim\left\|\varphi^{2} v\right\|_{L^{2}}
$$

So $I_{\mathbf{W}}$ can be bounded by

$$
I_{\mathbf{W}} \leq C\left\|\varphi^{2} v\right\|_{L^{2}}\left(\|\nabla w \otimes v\|_{L^{2}}+\|\varpi \times w\|_{L^{2}}+\|\varpi \times u+\omega \times w\|_{L^{\frac{6}{5}}}\right) .
$$

In summary, we conclude that for $-4 \leq t \leq 0$,

$$
\frac{\mathrm{d}}{\mathrm{d} t} \int \varphi^{4} \frac{|v|^{2}}{2} \mathrm{~d} x+\int \varphi^{4}|\nabla v|^{2} \mathrm{~d} x \leq I_{\Delta}+I_{\mathbf{R}}+I_{\mathbf{B}}+I_{\mathbf{L}}+I_{\mathbf{W}}
$$

with good estimates on each of the term on the right.

4.3. Proof of Proposition 4.1, First we check the integrability of each terms.

Lemma 4.4 (Integrability). Given conditions (1.4) and (1.5), we have

$$
\begin{aligned}
& \|u\|_{L_{t}^{p_{1}} L_{x}^{q_{3}}\left(Q_{2}\right)} \leq \eta \\
& \|\varphi \omega\|_{L_{t}^{p_{1}} L_{x}^{q_{1}}\left((-4,0) \times \mathbb{R}^{3}\right)} \leq \eta, \quad\|\varphi \omega\|_{L_{t}^{p_{2}} L_{x}^{q_{2}}\left((-4,0) \times \mathbb{R}^{3}\right)} \leq \eta, \\
& \|\nabla v\|_{L_{t}^{p_{1}} L_{x}^{q_{1}}\left((-4,0) \times \mathbb{R}^{3}\right)} \leq \eta, \quad\|\nabla v\|_{L_{t}^{p_{2}} L_{x}^{q_{2}}\left((-4,0) \times \mathbb{R}^{3}\right)} \leq \eta, \\
& \|v\|_{L_{t}^{p_{1}} L_{x}^{q_{3}}\left((-4,0) \times \mathbb{R}^{3}\right)} \leq \eta, \quad\|v\|_{L_{t}^{p_{2}} L_{x}^{q_{4}}\left((-4,0) \times \mathbb{R}^{3}\right)} \leq \eta, \\
& \|\nabla w\|_{L_{t}^{p_{1}} L_{x}^{q_{3}}\left((-4,0) \times \mathbb{R}^{3}\right)} \leq \eta, \\
& \|w\|_{L_{t}^{p_{1}} L_{x}^{q_{5}}\left((-4,0) \times \mathbb{R}^{3}\right)} \leq \eta \\
& \|\varpi\|_{L_{t}^{p_{1}} L_{x}^{q_{3}}\left((-4,0) \times \mathbb{R}^{3}\right)} \leq \eta, \quad\|\varpi\|_{L_{t}^{p_{2}} L_{x}^{q_{4}}\left((-4,0) \times \mathbb{R}^{3}\right)} \leq \eta .
\end{aligned}
$$

Proof. Integrability of $u$ is obtained by Sobolev embedding and that $\varphi u$ has average 0 . Integrability of $\varphi \omega$ is given. The remaining are consequences of Lemma 4.3 and Sobolev embedding. 
Proof of Proposition 4.1. We prove Proposition 4.1 using a Grönwall argument. Multiply (4.11) by an increasing smooth function $\psi_{1}(t)$ with $\psi_{1}(t)=0$ for $t \leq-2$, $\psi_{1}(t)=1$ for $t \geq 0$, we have

$$
\begin{aligned}
& \frac{\mathrm{d}}{\mathrm{d} t}\left(\psi_{1}(t) \int \varphi^{4} \frac{|v|^{2}}{2} \mathrm{~d} x\right)+\psi_{1}(t) \int \varphi^{4}|\nabla v|^{2} \mathrm{~d} x \\
& \quad=\psi_{1}^{\prime}(t) \int \varphi^{4} \frac{|v|^{2}}{2} \mathrm{~d} x+\psi_{1}(t)\left(I_{\Delta}+I_{\mathbf{R}}+I_{\mathbf{B}}+I_{\mathbf{L}}+I_{\mathbf{W}}\right) .
\end{aligned}
$$

Integrate from -4 to $t<0$ we have

$$
\begin{aligned}
& \psi_{1}(t) \int \varphi^{4} \frac{|v|^{2}}{2} \mathrm{~d} x+\int_{-2}^{t} \psi_{1}(s) \int \varphi^{4}|\nabla v|^{2} \mathrm{~d} x
\end{aligned}
$$

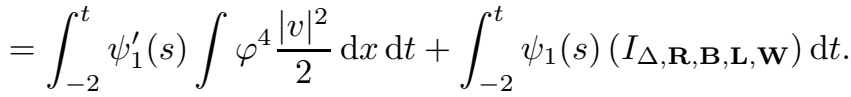

Because (4.6), (4.7), (4.8), (4.9), (4.10), and

$$
\left\|\varphi^{2} v\right\|_{L^{2}\left(B_{2}\right)},\left\|\varphi^{2} v\right\|_{L^{2}\left(B_{2}\right)}^{\frac{1}{3}} \leq C\left(1+\int \varphi^{4}|v|^{2} \mathrm{~d} x\right)
$$

we can conclude that

$$
\begin{gathered}
\frac{\mathrm{d}}{\mathrm{d} t}\left(\psi_{1}(t) \int \varphi^{4} \frac{|v|^{2}}{2} \mathrm{~d} x\right)+\psi_{1}(t) \int \varphi^{4}|\nabla v|^{2} \mathrm{~d} x \\
\leq C \Phi(t)\left(1+\psi_{1}(t) \int \varphi^{4} \frac{|v|^{2}}{2} \mathrm{~d} x\right),
\end{gathered}
$$

where

$$
\begin{aligned}
\Phi(t)= & \psi_{1}^{\prime}(t) \int \varphi^{4} \frac{|v|^{2}}{2} \mathrm{~d} x \\
& +\|v\|_{L^{2}}+\|u \otimes v\|_{L^{2}}+\|\omega \times u\|_{L^{\frac{6}{5}}\left(B_{2}\right)} \\
& +\|v\|_{L_{x}^{q_{3}}}^{\frac{2}{3}}\|\omega\|_{L_{x}^{q_{2}}\left(B_{2}\right)}+\|\nabla w \otimes v\|_{L^{2}} \\
& +\|\varpi \times w\|_{L^{2}}+\|\varpi \times u+\omega \times w\|_{L^{\frac{6}{5}}} \\
\leq \| & v\left\|_{L^{2}}^{2}+\right\| v\left\|_{L^{2}}+\right\| v\left\|_{L_{x}^{q_{4}}}\right\| u\left\|_{L_{x}^{q_{3}}\left(B_{2}\right)}+\right\| \omega\left\|_{L_{x}^{q_{2}}\left(B_{2}\right)}\right\| u \|_{L_{x}^{q_{3}}\left(B_{2}\right)} \\
& +\|v\|_{L_{x}^{q_{3}}}^{\frac{2}{3}}\|\omega\|_{L_{x}^{q_{2}}\left(B_{2}\right)}+\|\nabla w\|_{L_{x}^{q_{3}}}\|v\|_{L_{x}^{q_{4}}} \\
& +\|\varpi\|_{L_{x}^{q_{4}}}\|w\|_{L_{x}^{q_{3}}} \\
& +\|\varpi\|_{L_{x}^{q_{4}}}\|u\|_{L_{x}^{q_{3}}}+\|\omega\|_{L_{x}^{q_{2}}}\|w\|_{L_{x}^{q_{3}}} \\
\leq( & \left.\|v\|_{L_{x}^{q_{3}}}+\|v\|_{L_{x}^{q_{3}}}^{\frac{1}{2}}+\|u\|_{L_{x}^{q_{3}}\left(B_{2}\right)}+\|\nabla w\|_{L_{x}^{q_{3}}}+\|w\|_{L_{x}^{q_{3}}}\right) \\
& \times\left(\|v\|_{L_{x}^{q_{4}}}+\|v\|_{L_{x}^{q_{4}}}^{\frac{1}{2}}+\|\omega\|_{L_{x}^{q_{2}}}+\|\varpi\|_{L_{x}^{q_{4}}}\right)
\end{aligned}
$$

Here we used interpolation for $\|v\|_{L^{2}}^{2} \leq\|v\|_{L_{x}^{q_{3}}}\|v\|_{L_{x}^{q_{4}}}$. Therefore

$$
\begin{aligned}
\|\Phi\|_{L_{t}^{1}} \lesssim & \left\|\left(\|v\|_{L_{x}^{q_{3}}}+\|v\|_{L_{x}^{q_{3}}}^{\frac{1}{2}}+\|u\|_{L_{x}^{q_{3}}\left(B_{2}\right)}+\|\nabla w\|_{L_{x}^{q_{3}}}+\|w\|_{L_{x}^{q_{3}}}\right)\right\|_{L_{t}^{p_{1}}} \\
\times & \left\|\left(\|v\|_{L_{x}^{q_{4}}}+\|v\|_{L_{x}^{q_{4}}}^{\frac{1}{2}}+\|\omega\|_{L_{x}^{q_{2}}}+\|\varpi\|_{L_{x}^{q_{4}}}\right)\right\|_{L_{t}^{p_{2}}} \leq \eta .
\end{aligned}
$$


By a Grönwall's lemma, we conclude that for every $-4 \leq t \leq 0$,

$$
1+\psi_{1}(t) \int \varphi^{4} \frac{|v|^{2}}{2} \mathrm{~d} x+\int_{-4}^{t} \psi_{1}(t) \int \varphi^{4}|\nabla v|^{2} \mathrm{~d} x \leq e^{\int_{-4}^{t} C \Phi(s) \mathrm{d} s} \leq e^{C \eta} .
$$

Therefore by taking the sup over $-1 \leq t \leq 0$ and $t=0$ respectively, we conclude

$$
\sup _{-1 \leq t \leq 0} \int|v(t)|^{2} \mathrm{~d} x \leq \eta, \quad \int_{Q_{1}}|\nabla v|^{2} \mathrm{~d} x \mathrm{~d} t \leq \eta .
$$

\section{Local Study: Part Two, De Giorgi Iteration}

In this section, we derive the boundedness of $v$ in $Q_{\frac{1}{2}}$ which is the following.

Proposition 5.1. Let $v$ solves (4.4). If (4.1) holds for sufficiently small $\eta$, and we have integrability bounds in Lemma 4.4, then we have

$$
\|v\|_{L^{\infty}\left(Q_{\frac{1}{2}}\right)}=\sup _{t \in(-1,0)}\|v(t)\|_{L^{\infty}\left(B_{\frac{1}{2}}\right)} \leq 1 .
$$

The proof uses De Giorgi technique and the truncation method. First, we set dyadically shrinking radius,

$$
r_{k}^{b}=\frac{1}{2}\left(1+8^{-k}\right), \quad r_{k}^{\natural}=\frac{1}{2}\left(1+2 \times 8^{-k}\right), \quad r_{k}^{\sharp}=\frac{1}{2}\left(1+4 \times 8^{-k}\right) .
$$

Then we define dyadically shrinking cylinder $Q_{k}$ 's,

$$
\begin{aligned}
& T_{k}^{b}=r_{k}^{b^{2}}, \quad B_{k}^{b}=B_{r_{k}^{b}}(0), \quad Q_{k}^{b}=\left(-T_{k}^{b}, 0\right) \times B_{k}^{b}, \\
& T_{k}^{\natural}=r_{k}^{\natural}, \quad \quad B_{k}^{\natural}=B_{r_{k}^{\natural}}(0), \quad Q_{k}^{\natural}=\left(-T_{k}^{\natural}, 0\right) \times B_{k}^{\natural}, \\
& T_{k}^{\sharp}=r_{k}^{\sharp}, \quad \quad B_{k}^{\sharp}=B_{r_{k}^{\sharp}}(0), \quad Q_{k}^{\sharp}=\left(-T_{k}^{\sharp}, 0\right) \times B_{k}^{\sharp} .
\end{aligned}
$$

We also introduce positive smooth space-time cut-off functions $\rho_{k}$ and $\rho_{k}^{\sharp}$ with

$$
\mathbf{1}_{Q_{k}^{b}} \leq \rho_{k} \leq \mathbf{1}_{Q_{k}^{\natural}}, \quad \mathbf{1}_{Q_{k}^{\sharp}} \leq \rho_{k}^{\sharp} \leq \mathbf{1}_{Q_{k-1}^{b}} .
$$

Then, let $c_{k}$ denote a sequence of rising energy level,

$$
\begin{aligned}
& c_{k}=1-2^{-k}, \quad v_{k}=\left(|v|-c_{k}\right)_{+}, \quad \beta_{k}=\frac{v_{k}}{|v|}, \\
& \Omega_{k}=\left\{v_{k}>0\right\}, \quad \mathbf{1}_{k}=\mathbf{1}_{\Omega_{k}}, \quad \alpha_{k}=1-\beta_{k} .
\end{aligned}
$$

We define analogous of vector derivative $d_{k}$ and energy quantity $U_{k}$ :

$$
\begin{aligned}
d_{k}^{2} & =\mathbf{1}_{k}\left(\alpha_{k}|\nabla| v \|^{2}+\beta_{k}|\nabla v|^{2}\right), \\
U_{k} & =\left\|v_{k}\right\|_{L^{\infty}\left(-T_{k}^{b}, 0 ; L^{2}\left(B_{k}^{b}\right)\right)}^{2}+\left\|d_{k}\right\|_{L^{2}\left(Q_{k}^{b}\right)}^{2} .
\end{aligned}
$$

We have the following truncation estimates.

\section{Lemma 5.2.}

$$
\begin{aligned}
\alpha_{k} v \leq c_{k} & \leq 1, \\
\left\|\beta_{k} v\right\|_{L_{t}^{\infty} L_{x}^{2} \cap L_{t}^{2} \dot{H}_{x}^{1}\left(Q_{k-1}^{b}\right)}^{2} & \leq 9 U_{k-1}, \\
\left\|\mathbf{1}_{k}\right\|_{L_{t}^{\infty} L_{x}^{2} \cap L_{t}^{2} L_{x}^{6}\left(Q_{k-1}^{b}\right)} & \leq C^{k} U_{k-1} .
\end{aligned}
$$


Proof. The first estimate follows from the definition. By Lemma 4 in Vas07, we have $\left|\nabla v_{k}\right| \leq d_{k}$ and $\left|\nabla\left(\beta_{k} v\right)\right| \leq 3 d_{k}$. Moreover, since $|\nabla| v|| \leq|\nabla v|^{2}$, we see $d_{k} \leq d_{k-1}$, as $v_{k}$ and $\beta_{k}$ are monotonously decreasing. So

$$
\left\|\nabla\left(\beta_{k} v\right)\right\|_{L^{2}\left(Q_{k-1}^{b}\right)} \leq 3\left\|d_{k}\right\|_{L^{2}\left(Q_{k-1}^{b}\right)} \leq 3\left\|d_{k-1}\right\|_{L^{2}\left(Q_{k-1}^{b}\right)} .
$$

Moreover, the truncation gives $\left|\beta_{k} v\right|+2^{-k} \mathbf{1}_{k}=v_{k}+2^{-k} \mathbf{1}_{k}=\mathbf{1}_{k} v_{k-1}$, so

$$
\begin{aligned}
\left\|\beta_{k} v\right\|_{L_{t}^{\infty} L_{x}^{2}\left(Q_{k-1}^{b}\right)} & \leq\left\|v_{k-1}\right\|_{L_{t}^{\infty} L_{x}^{2}\left(Q_{k-1}^{b}\right)}, \\
2^{-k}\left\|\mathbf{1}_{k}\right\|_{L_{t}^{\infty} L_{x}^{2}\left(Q_{k-1}^{b}\right)} & \leq\left\|v_{k-1}\right\|_{L_{t}^{\infty} L_{x}^{2}\left(Q_{k-1}^{b}\right)}, \\
2^{-k}\left\|\mathbf{1}_{k}\right\|_{L_{t}^{2} L_{x}^{6}\left(Q_{k-1}^{b}\right)} & \leq\left\|v_{k-1}\right\|_{L_{t}^{2} L_{x}^{6}\left(Q_{k-1}^{b}\right)} \\
& \leq\left\|v_{k-1}\right\|_{L_{t}^{\infty} L_{x}^{2}\left(Q_{k-1}^{b}\right)}+\left\|\nabla v_{k-1}\right\|_{L^{2}\left(Q_{k-1}^{b}\right)} \\
& \leq\left\|v_{k-1}\right\|_{L_{t}^{\infty} L_{x}^{2}\left(Q_{k-1}^{b}\right)}+\left\|d_{k-1}\right\|_{L^{2}\left(Q_{k-1}^{b}\right)} .
\end{aligned}
$$

Corollary 5.3 (Nonlinearization). If $f \in L_{t}^{p} L_{x}^{q}\left(Q_{k-1}\right)$, with

$$
\frac{1}{p}+\gamma\left(\frac{\theta}{2}+\frac{1-\theta}{\infty}\right)=1, \quad \frac{1}{q}+\gamma\left(\frac{\theta}{6}+\frac{1-\theta}{2}\right)=1,
$$

for some $0 \leq \theta \leq 1,0<\sigma \leq \gamma$, then uniformly in $\sigma$,

$$
\int_{Q_{k-1}^{b}}\left|\beta_{k} v\right|^{\sigma}|f| \mathrm{d} x \mathrm{~d} t \leq C^{k}\|f\|_{L_{t}^{p} L_{x}^{q}\left(Q_{k-1}\right)} U_{k-1}^{\frac{\gamma}{2}} .
$$

Proof. By interpolation,

$$
\left\|\beta_{k} v\right\|,\left\|\mathbf{1}_{k}\right\|_{L_{t}^{p_{\theta}} L_{x}^{q_{\theta}\left(Q_{k-1}\right)}} \leq U_{k-1}^{\frac{1}{2}}
$$

where

$$
\frac{1}{p_{\theta}}=\frac{\theta}{2}+\frac{1-\theta}{\infty}, \quad \frac{1}{q_{\theta}}=\frac{\theta}{6}+\frac{1-\theta}{2} .
$$

Therefore, using Hölder's inequality,

$$
\int_{Q_{k-1}}\left|\beta_{k} v\right|^{\sigma}|f| \mathrm{d} x \mathrm{~d} t \leq\|f\|_{L_{t}^{p} L_{x}^{q}}\left\|\beta_{k} v\right\|_{L_{t}^{p_{\theta}} L_{x}^{q_{\theta}}}^{\sigma}\left\|\mathbf{1}_{k}\right\|_{L_{t}^{p_{\theta}} L_{x}^{q_{\theta}}}^{\gamma-\sigma} \leq\|f\|_{L_{t}^{p} L_{x}^{q}} U_{k-1}^{\frac{\gamma}{2}} .
$$

First, we recall the following identities from Vas07.

$$
\begin{aligned}
& \alpha_{k} v \cdot \partial_{\bullet} v=\partial_{\bullet}\left(\frac{|v|^{2}-v_{k}^{2}}{2}\right), \\
& \alpha_{k} v \cdot \Delta v=\Delta\left(\frac{|v|^{2}-v_{k}^{2}}{2}\right)+d_{k}^{2}-|\nabla v|^{2} .
\end{aligned}
$$

Since $\alpha_{k} v$ is bounded, we can multiply equation (4.4) by $\alpha_{k} v$ and obtain

$$
\begin{aligned}
& \partial_{t}\left(\frac{|v|^{2}-v_{k}^{2}}{2}\right)+\alpha_{k} v \cdot \nabla \mathbf{R}(u \otimes v) \\
& \quad=\Delta\left(\frac{|v|^{2}-v_{k}^{2}}{2}\right)+d_{k}^{2}-|\nabla v|^{2}+\alpha_{k} v \cdot(\mathbf{B}+\mathbf{L}+\mathbf{W}) .
\end{aligned}
$$


using (5.1) and (5.2). Denote $\mathbf{C}_{v}=\mathbf{B}+\mathbf{L}+\mathbf{W}$. Subtracting (5.3) from (4.5), we have

$$
\partial_{t} \frac{v_{k}^{2}}{2}+d_{k}^{2}+\operatorname{div}(v \mathbf{R}(u \otimes v))-\alpha_{k} v \cdot \nabla \mathbf{R}(u \otimes v) \leq \Delta \frac{v_{k}^{2}}{2}+\beta_{k} v \cdot \mathbf{C}_{v} .
$$

Multiply by $\rho_{k}$, then integrate in space and from $\sigma$ to $\tau$ in time,

$$
\begin{aligned}
& {\left[\int \rho_{k} \frac{v_{k}^{2}}{2} \mathrm{~d} x\right]_{\sigma}^{\tau}+\int_{\sigma}^{\tau} \int \rho_{k} d_{k}^{2} \mathrm{~d} x \mathrm{~d} t} \\
& \leq \int_{\sigma}^{\tau} \int\left(\partial_{t} \rho_{k}+\Delta \rho_{k}\right) \frac{v_{k}^{2}}{2} \mathrm{~d} x \mathrm{~d} t-\int_{\sigma}^{\tau} \int \rho_{k} \operatorname{div}(v \mathbf{R}(u \otimes v)) \mathrm{d} x \mathrm{~d} t \\
& \quad+\int_{\sigma}^{\tau} \int \rho_{k} \alpha_{k} v \cdot \nabla \mathbf{R}(u \otimes v) \mathrm{d} x \mathrm{~d} t+\int_{\sigma}^{\tau} \int \rho_{k} \beta_{k} v \cdot \mathbf{C}_{v} \mathrm{~d} x \mathrm{~d} t
\end{aligned}
$$

Take the sup over $\tau>-T_{k}^{b}$, and set $\sigma<-T_{k-1}^{b}$, we obtain

$$
\begin{aligned}
& U_{k} \leq \sup _{\tau \in\left(-T_{k}^{b}, 0\right)} \int \rho_{k} \frac{v_{k}^{2}}{2} \mathrm{~d} x+\int_{-T_{k-1}^{b}}^{0} \int \rho_{k} d_{k}^{2} \mathrm{~d} x \mathrm{~d} t \\
& \leq C^{k} \int_{Q_{k}^{\natural}} v_{k}^{2} \mathrm{~d} x \mathrm{~d} t+\sup _{\tau \in\left(-T_{k}^{b}, 0\right)}\{ \int_{-T_{k}^{\natural}}^{\tau} \int \rho_{k} \alpha_{k} v \cdot \nabla \mathbf{R}(u \otimes v) \mathrm{d} x \mathrm{~d} t \\
&-\int_{-T_{k}^{\natural}}^{\tau} \int \rho_{k} \operatorname{div}(v \mathbf{R}(u \otimes v)) \mathrm{d} x \mathrm{~d} t \\
&\left.+\int_{-T_{k}^{\natural}}^{\tau} \int \rho_{k} \beta_{k} v \cdot \mathbf{C}_{v} \mathrm{~d} x \mathrm{~d} t\right\} .
\end{aligned}
$$

Using Corollary [5.3, the first one is bounded by

$$
\int_{Q_{k}^{\natural}} v_{k}^{2} \mathrm{~d} x \mathrm{~d} s \leq \int_{Q_{k-1}^{b}}\left|\beta_{k} v\right|^{2} \mathrm{~d} x \mathrm{~d} s \leq U_{k-1}^{\frac{5}{3}} .
$$

Now let's deal with the last few terms. For simplicity, we use $\iint \mathrm{d} x \mathrm{~d} t$ to denote $\int_{-T_{k}^{\natural}}^{\tau} \int_{\mathbb{R}^{3}} \mathrm{~d} x \mathrm{~d} t$ in the rest of this section.

5.1. Highest Order Nonlinear Term. Define three trilinear forms,

$$
\begin{aligned}
\mathbf{T}_{\circ}\left[v_{1}, v_{2}, v_{3}\right] & =\iint \rho_{k} \operatorname{div}\left(v_{1} \mathbf{R}\left(v_{2} \otimes v_{3}\right)\right) \mathrm{d} x \mathrm{~d} t, \\
\mathbf{T}_{\nabla}\left[v_{1}, v_{2}, v_{3}\right] & =\iint \rho_{k} v_{1} \cdot \nabla \mathbf{R}\left(v_{2} \otimes v_{3}\right) \mathrm{d} x \mathrm{~d} t, \\
\mathbf{T}_{\text {div }}\left[v_{1}, v_{2}, v_{3}\right] & =\iint \rho_{k} \operatorname{div} v_{1} \mathbf{R}\left(v_{2} \otimes v_{3}\right) \mathrm{d} x \mathrm{~d} t .
\end{aligned}
$$

They are symmetric on $v_{2}, v_{3}$ positions. When we have enough integrability, that is, when

$$
\left|\nabla v_{1}\right|\left|v_{2}\right|\left|v_{3}\right|,\left|v_{1}\right|\left|\nabla v_{2}\right|\left|v_{3}\right|,\left|v_{1}\right|\left|v_{2}\right|\left|\nabla v_{3}\right| \in L_{t, x}^{1}
$$

we have Leibniz rule

$$
\mathbf{T}_{\circ}=\mathbf{T}_{\nabla}+\mathbf{T}_{\text {div }}
$$


The goal is to estimate the first two double integrals in (5.4),

$$
\begin{aligned}
& \iint \rho_{k} \alpha_{k} v \cdot \nabla \mathbf{R}(u \otimes v) \mathrm{d} x \mathrm{~d} t-\iint \rho_{k} \operatorname{div}(v \mathbf{R}(u \otimes v)) \mathrm{d} x \mathrm{~d} t \\
& =\mathbf{T}_{\nabla}\left[\alpha_{k} v, u, v\right]-\mathbf{T}_{\circ}[v, u, v] .
\end{aligned}
$$

We first separate $w \otimes v$ from $u \otimes v$, and we will have

$$
\begin{aligned}
\mathbf{T}_{\nabla}\left[\alpha_{k} v, w, v\right]-\mathbf{T}_{\circ}[v, w, v] & =\mathbf{T}_{\nabla}\left[\alpha_{k} v, w, v\right]-\mathbf{T}_{\nabla}[v, w, v]-\mathbf{T}_{\mathrm{div}}[v, w, v] \\
& =-\mathbf{T}_{\nabla}\left[\beta_{k} v, w, v\right] \\
& =-\iint \rho_{k} \beta_{k} v \cdot \nabla \mathbf{R}(w \otimes v) \mathrm{d} x \mathrm{~d} t .
\end{aligned}
$$

Denote $-\nabla \mathbf{R}(w \otimes v)=: \mathbf{W}_{2}$ and we will deal with it later. The remaining $(u-w) \otimes v$ can be separated into interior part and exterior part,

$$
(u-w) \otimes v=\rho_{k}^{\sharp} v \otimes v+\left(1-\rho_{k}^{\sharp}\right)(u-w) \otimes v .
$$

The exterior part is bounded and smooth in space over the support of $\rho_{k}$.

$$
\begin{aligned}
\left\|\rho_{k} \mathbf{R}\left(\left(1-\rho_{k}^{\sharp}\right)(u-w) \otimes v\right)\right\|_{L_{t}^{p_{3}} C_{x}^{\infty}} & \leq C\|(u-w) \otimes v\|_{L_{t}^{p_{3}} L_{x}^{2}} \\
& \leq C\|u-w\|_{L_{t}^{p_{1}} L_{x}^{q_{3}}\left(Q_{2}\right)}\|v\|_{L_{t}^{p_{2}} L_{x}^{q_{4}}} \leq \eta .
\end{aligned}
$$

Here, we denote

$$
\frac{1}{p_{3}}=\frac{1}{p_{1}}+\frac{1}{p_{2}}<1
$$

Therefore we can use Leibniz rule similar as $w$ and

$$
\begin{aligned}
& \mathbf{T}_{\nabla}\left[\alpha_{k} v,\left(1-\rho_{k}^{\sharp}\right)(u-w), v\right]-\mathbf{T}_{\circ}\left[v,\left(1-\rho_{k}^{\sharp}\right)(u-w), v\right] \\
& =\mathbf{T}_{\nabla}\left[\alpha_{k} v,\left(1-\rho_{k}^{\sharp}\right)(u-w), v\right]-\mathbf{T}_{\nabla}\left[v,\left(1-\rho_{k}^{\sharp}\right)(u-w), v\right] \\
& =-\mathbf{T}_{\nabla}\left[\beta_{k} v,\left(1-\rho_{k}^{\sharp}\right)(u-w), v\right] \\
& =-\iint \rho_{k} \beta_{k} v \cdot \nabla \mathbf{R}\left(v \otimes\left(1-\rho_{k}^{\sharp}\right)(u-w)\right) \mathrm{d} x \mathrm{~d} t \\
& \leq C^{k} U_{k-1}^{\frac{5}{3}-\frac{2}{3 p_{3}}}
\end{aligned}
$$


by nonlinearization Corollary 5.3 The interior part is

$$
\begin{aligned}
\mathbf{T}_{\nabla}[ & \left.\alpha_{k} v, \rho_{k}^{\sharp} v, v\right]-\mathbf{T}_{\circ}\left[v, \rho_{k}^{\sharp} v, v\right] \\
=\mathbf{T}_{\nabla} & {\left[\alpha_{k} v, \rho_{k}^{\sharp} \beta_{k} v, \beta_{k} v\right]+2 \mathbf{T}_{\nabla}\left[\alpha_{k} v, \rho_{k}^{\sharp} \alpha_{k} v, \beta_{k} v\right] } \\
& +\mathbf{T}_{\nabla}\left[\alpha_{k} v, \rho_{k}^{\sharp} \alpha_{k} v, \alpha_{k} v\right]-\mathbf{T}_{\circ}\left[v, \rho_{k}^{\sharp} v, v\right] \\
= & \mathbf{T}_{\nabla}\left[\alpha_{k} v, \rho_{k}^{\sharp} \beta_{k} v, \beta_{k} v\right] \\
& +2 \mathbf{T}_{\circ}\left[\alpha_{k} v, \rho_{k}^{\sharp} \alpha_{k} v, \beta_{k} v\right]-2 \mathbf{T}_{\operatorname{div}}\left[\alpha_{k} v, \rho_{k}^{\sharp} \alpha_{k} v, \beta_{k} v\right] \\
& +\mathbf{T}_{\circ}\left[\alpha_{k} v, \rho_{k}^{\sharp} \alpha_{k} v, \alpha_{k} v\right]-\mathbf{T}_{\operatorname{div}}\left[\alpha_{k} v, \rho_{k}^{\sharp} \alpha_{k} v, \alpha_{k} v\right] \\
& -\mathbf{T}_{\circ}\left[v, \rho_{k}^{\sharp} v, v\right] \\
= & \mathbf{T}_{\nabla}\left[\alpha_{k} v, \rho_{k}^{\sharp} \beta_{k} v, \beta_{k} v\right] \\
& +2 \mathbf{T}_{\operatorname{div}}\left[\beta_{k} v, \rho_{k}^{\sharp} \alpha_{k} v, \beta_{k} v\right]+\mathbf{T}_{\operatorname{div}}\left[\beta_{k} v, \rho_{k}^{\sharp} \alpha_{k} v, \alpha_{k} v\right] \\
& +2 \mathbf{T}_{\circ}\left[\alpha_{k} v, \rho_{k}^{\sharp} \alpha_{k} v, \beta_{k} v\right]+\mathbf{T}_{\circ}\left[\alpha_{k} v, \rho_{k}^{\sharp} \alpha_{k} v, \alpha_{k} v\right] \\
& -\mathbf{T}_{\circ}\left[v, \rho_{k}^{\sharp} v, v\right] \\
= & \mathbf{T}_{\nabla}\left[\alpha_{k} v, \rho_{k}^{\sharp} \beta_{k} v, \beta_{k} v\right]+\mathbf{T}_{\mathrm{div}}\left[\beta_{k} v, \rho_{k}^{\sharp} \alpha_{k} v,\left(\beta_{k}+1\right) v\right] \\
& -\mathbf{T}_{\circ}\left[\alpha_{k} v, \rho_{k}^{\sharp} \beta_{k} v, \beta_{k} v\right]-\mathbf{T}_{\circ}\left[\beta_{k} v, \rho_{k}^{\sharp} v, v\right] .
\end{aligned}
$$

Notice that the boundedness of $\alpha_{k} v$ guarantees enough integrability to switch between trilinear forms. Then

$$
\begin{gathered}
\left|\mathbf{T}_{\nabla}\left[\alpha_{k} v, \rho_{k}^{\sharp} \beta_{k} v, \beta_{k} v\right]\right|,\left|\mathbf{T}_{\mathrm{div}}\left[\beta_{k} v, \rho_{k}^{\sharp} \alpha_{k} v,\left(\beta_{k}+1\right) v\right]\right| \\
\lesssim\left\|\nabla\left(\beta_{k} v\right)\right\|_{L^{2}\left(Q_{k-1}\right)} U_{k-1}^{\frac{5}{6}} \leq U_{k-1}^{\frac{4}{3}}, \\
\left|\mathbf{T}_{\circ}\left[\alpha_{k} v, \rho_{k}^{\sharp} \beta_{k} v, \beta_{k} v\right]\right|,\left|\mathbf{T}_{\circ}\left[\beta_{k} v, \rho_{k}^{\sharp} v, v\right]\right| \lesssim U_{k-1}^{\frac{5}{3}} .
\end{gathered}
$$

In conclusion,

$$
\begin{gathered}
\mid \iint \rho_{k} \alpha_{k} v \cdot \nabla \mathbf{R}(u \otimes v) \mathrm{d} x \mathrm{~d} t-\iint \rho_{k} \operatorname{div}(v \mathbf{R}(u \otimes v)) \mathrm{d} x \mathrm{~d} t \\
-\iint \rho_{k} \beta_{k} v \cdot \mathbf{W}_{2} \mathrm{~d} x \mathrm{~d} t \mid \lesssim C^{k} U_{k-1}^{\min \left\{\frac{4}{3}, \frac{5}{3}-\frac{2}{3} p_{3}\right\}} .
\end{gathered}
$$

5.2. Lower Order Terms. For the bilinear and linear term, recall that inside $B_{1}$,

$$
\begin{aligned}
& \mathbf{B}=-\operatorname{curl} \Delta^{-1}(\nabla \varphi \times(\omega \times u)), \\
& \mathbf{L}=\operatorname{curl} \Delta^{-1}(2 \operatorname{div}(\nabla \varphi \otimes \omega)-(\Delta \varphi) \omega) .
\end{aligned}
$$

Therefore,

$$
\begin{aligned}
&\left\|\rho_{k} \mathbf{B}\right\|_{L_{t}^{p_{3}} L_{x}^{\infty}} \leq\|\omega \times u\|_{L_{t}^{p_{3}} L_{x}^{\frac{6}{5}}\left(Q_{2}\right)} \leq\|u\|_{L_{t}^{p_{1}} L_{x}^{q_{3}}}\|\omega\|_{L_{t}^{p_{2}} L_{x}^{q_{2}}} \leq \eta \\
&\left\|\rho_{k} \mathbf{L}\right\|_{L_{t}^{p_{2}} L_{x}^{\infty}} \leq\|\omega\|_{L_{t}^{p_{2}} L_{x}^{q_{2}}\left(Q_{2}\right)} \leq \eta
\end{aligned}
$$

Thus

$$
\begin{aligned}
& \iint \mathbf{B} \cdot \rho_{k} \beta_{k} v \mathrm{~d} x \mathrm{~d} t \leq C^{k} U_{k-1}^{\frac{5}{3}-\frac{2}{3 p_{3}}} \\
& \iint \mathbf{L} \cdot \rho_{k} \beta_{k} v \mathrm{~d} x \mathrm{~d} t \leq C^{k} U_{k-1}^{\frac{5}{3}-\frac{2}{3 p_{2}}}
\end{aligned}
$$


5.3. W Terms. Finally, let us deal with

$$
\mathbf{W}+\mathbf{W}_{2}=-\omega \times w+\frac{1}{2} \mathbb{P}_{\nabla}(\varpi \times u+\omega \times w)-\nabla \mathbf{R}(w \otimes v)
$$

Here $\nabla \mathbf{R}=\frac{1}{2} \nabla \operatorname{tr}-\mathbb{P}_{\nabla}$ div, so

$$
\begin{aligned}
\nabla \mathbf{R}(w \otimes v)= & \frac{1}{2} \nabla(w \cdot v)-\mathbb{P}_{\nabla} \operatorname{div}(v \otimes w) \\
= & \frac{1}{2}(w \cdot \nabla v+v \cdot \nabla w+w \times \operatorname{curl} v+v \times \operatorname{curl} w)-\mathbb{P}_{\nabla}(v \cdot \nabla w) \\
= & \frac{1}{2}(w \cdot \nabla v-v \cdot \nabla w)+\mathbb{P}_{\operatorname{curl}}(v \cdot \nabla w) \\
& \quad+\frac{1}{2}(w \times \operatorname{curl} v+v \times \operatorname{curl} w) \\
\nabla \mathbf{R}(w \otimes v)= & \mathbb{P}_{\nabla}(\nabla \mathbf{R}(w \otimes v)) \\
= & \frac{1}{2} \mathbb{P}_{\nabla}(w \cdot \nabla v-v \cdot \nabla w)+\frac{1}{2} \mathbb{P}_{\nabla}(w \times \operatorname{curl} v+v \times \operatorname{curl} w) \\
= & \frac{1}{2} \mathbb{P}_{\nabla}(\operatorname{curl}(v \times w)-v \operatorname{div} w+w \operatorname{div} v) \\
& \quad+\frac{1}{2} \mathbb{P}_{\nabla}(w \times \operatorname{curl} v+v \times \operatorname{curl} w) \\
= & -\frac{1}{2} \mathbb{P}_{\nabla}(v(u \cdot \nabla \varphi))+\frac{1}{2} \mathbb{P}_{\nabla}(w \times \operatorname{curl} v+v \times \operatorname{curl} w) .
\end{aligned}
$$

Hence

$$
\begin{aligned}
\mathbf{W}+\mathbf{W}_{2}=- & \omega \times w+\frac{1}{2} \mathbb{P}_{\nabla}(v(u \cdot \nabla \varphi)) \\
& +\frac{1}{2} \mathbb{P}_{\nabla}(\varpi \times u+\omega \times w+\operatorname{curl} v \times w+\operatorname{curl} w \times v) .
\end{aligned}
$$

Again, we separate $\mathbf{W}+\mathbf{W}_{2}$ into exterior and interior part, with

$$
\mathbf{W}+\mathbf{W}_{2}=\mathbf{W}_{\text {ext }}+\mathbf{W}_{\text {int }}
$$


where

$$
\begin{aligned}
\mathbf{W}_{\text {ext }}=- & \left(1-\rho_{k}^{\sharp}\right) \omega \times w+\frac{1}{2} \mathbb{P}_{\nabla}(v(u \cdot \nabla \varphi)) \\
& +\frac{1}{2} \mathbb{P}_{\nabla}\left(\left(1-\rho_{k}^{\sharp}\right)(\varpi \times u+\omega \times w+\operatorname{curl} v \times w+\operatorname{curl} w \times v)\right), \\
\mathbf{W}_{\text {int }}=- & \rho_{k}^{\sharp} \omega \times w \\
& +\frac{1}{2} \mathbb{P}_{\nabla}\left(\rho_{k}^{\sharp}(\varpi \times u+\omega \times w+\operatorname{curl} v \times w+\operatorname{curl} w \times v)\right) \\
=- & \rho_{k}^{\sharp} \operatorname{curl} v \times w-\rho_{k}^{\sharp} \varpi \times w \\
& +\frac{1}{2} \mathbb{P}_{\nabla}\left(\rho_{k}^{\sharp}(\varpi \times u+\operatorname{curl} w \times v+\varpi \times w)\right) \\
& +\frac{1}{2} \mathbb{P}_{\nabla}\left(\rho_{k}^{\sharp}(\omega \times w+\operatorname{curl} v \times w-\varpi \times w)\right) \\
=- & \rho_{k}^{\sharp} \operatorname{curl} v \times w-\rho_{k}^{\sharp} \varpi \times w \\
& +\mathbb{P}_{\nabla}\left(\rho_{k}^{\sharp} \varpi \times u\right)+\mathbb{P}_{\nabla}\left(\rho_{k}^{\sharp} \operatorname{curl} v \times w\right) \\
= & \mathbb{P}_{\operatorname{curl}}\left(\rho_{k}^{\sharp} \operatorname{curl} v \times w\right)-\mathbb{P}_{\operatorname{curl}}\left(\rho_{k}^{\sharp} \varpi \times w\right)+\mathbb{P}_{\nabla}\left(\rho_{k}^{\sharp} \varpi \times v\right) .
\end{aligned}
$$

Similar as bilinear terms, $\rho_{k} \mathbf{W}_{\text {ext }}$ is small in $L_{t}^{p_{3}} L_{x}^{\infty}$. Among the three terms in $\mathbf{W}_{\text {int }}, \rho_{k}^{\sharp} \varpi \times w$ is bounded in $L_{t}^{p_{3}} L_{x}^{\infty}$, and $\rho_{k}^{\sharp} \varpi$ is in $L_{t}^{p_{2}} L_{x}^{\infty}$. Finally, for the first term,

$$
\begin{aligned}
\mathbb{P}_{\text {curl }}\left(\operatorname{curl} v \times \rho_{k}^{\sharp} w\right) & =-\mathbb{P}_{\text {curl }}\left(\operatorname{curl} \rho_{k}^{\sharp} w \times v\right)+\mathbb{P}_{\text {curl }}\left(v \cdot \nabla \rho_{k}^{\sharp} w+\rho_{k}^{\sharp} w \cdot \nabla v\right), \\
\mathbb{P}_{\text {curl }}\left(\rho_{k}^{\sharp} w \cdot \nabla v\right) & =\mathbb{P}_{\text {curl }}\left(\operatorname{curl}\left(v \times \rho_{k}^{\sharp} w\right)+v \cdot \nabla \rho_{k}^{\sharp} w-v \operatorname{div} \rho_{k}^{\sharp} w\right) \\
& =\operatorname{curl}\left(v \times \rho_{k}^{\sharp} w\right)+\mathbb{P}_{\operatorname{curl}}\left(v \cdot \nabla \rho_{k}^{\sharp} w-v \operatorname{div} \rho_{k}^{\sharp} w\right), \\
\operatorname{curl}\left(v \times \rho_{k}^{\sharp} w\right) & =v \operatorname{div} \rho_{k}^{\sharp} w+\rho_{k}^{\sharp} w \cdot \nabla v-v \cdot \nabla \rho_{k}^{\sharp} w .
\end{aligned}
$$

Every term is a product of $v$ and $\nabla \rho_{k}^{\sharp} w$ (possibly with a Riesz transform) except $\rho_{k}^{\sharp} w \cdot \nabla v$. Because in $\Omega_{k}, \nabla|v|=\nabla v_{k}$ are the same, we have

$$
\begin{aligned}
\int \rho_{k} \beta_{k} v \cdot\left(\rho_{k}^{\sharp} w \cdot \nabla\right) v \mathrm{~d} x & =\int \rho_{k} \beta_{k}(w \cdot \nabla) \frac{|v|^{2}}{2} \mathrm{~d} x \\
& =\int \rho_{k} \beta_{k}|v|(w \cdot \nabla)|v| \mathrm{d} x \\
& =\int \rho_{k} v_{k}(w \cdot \nabla) v_{k} \mathrm{~d} x \\
& =\int \rho_{k}(w \cdot \nabla) \frac{v_{k}^{2}}{2} \mathrm{~d} x \\
& =-\int \frac{v_{k}^{2}}{2} \operatorname{div}\left(\rho_{k} w\right) \mathrm{d} x .
\end{aligned}
$$


Therefore, every term of $\mathbb{P}_{\text {curl }}\left(\operatorname{curl} v \times \rho_{k}^{\sharp} w\right)$ is a product of $v$ and $\nabla \rho_{k} w$ or $\nabla \rho_{k}^{\sharp} w$. Inside $B_{1}, w \in L_{t}^{p_{1}} C_{x}^{\infty}$. In conclusion,

$$
\begin{array}{r}
\iint \rho_{k} \beta_{k} v \cdot \mathbf{W}_{\text {ext }} \mathrm{d} x \mathrm{~d} t \leq C^{k} U_{k-1}^{\frac{5}{3}-\frac{2}{3 p_{3}},} \\
\iint \rho_{k} \beta_{k} v \cdot \mathbb{P}_{\text {curl }}\left(\rho_{k}^{\sharp} \operatorname{curl} v \times w\right) \mathrm{d} x \mathrm{~d} t \leq C^{k} U_{k-1}^{\frac{5}{3}-\frac{2}{3 p_{1}},}, \\
\iint \rho_{k} \beta_{k} v \cdot \mathbb{P}_{\text {curl }}\left(\rho_{k}^{\sharp} \varpi \times w\right) \mathrm{d} x \mathrm{~d} t \leq C^{k} U_{k-1}^{\frac{5}{3}-\frac{2}{3 p_{3}},} \\
\iint \rho_{k} \beta_{k} v \cdot \mathbb{P}_{\nabla}\left(\rho_{k}^{\sharp} \varpi \times v\right) \mathrm{d} x \mathrm{~d} t \leq C^{k} U_{k-1}^{\frac{5}{3}-\frac{2}{3 p_{2}}} .
\end{array}
$$

So the sum is bounded in

$$
\iint \rho_{k} \beta_{k} v \cdot\left(\mathbf{W}+\mathbf{W}_{2}\right) \mathrm{d} x \mathrm{~d} t=\iint \rho_{k} \beta_{k} v \cdot\left(\mathbf{W}_{\text {int }}+\mathbf{W}_{\text {ext }}\right) \mathrm{d} x \mathrm{~d} t \leq C^{k} U_{k-1}^{\frac{5}{3}-\frac{2}{3 p_{3}}}
$$

provided $U_{k-1}<1$.

\subsection{Proof of Proposition 5.1.}

Proof of Proposition 5.1. Coming back to (5.4), by estimates (5.5) on the first term, (5.6) on the trilinear terms, (5.7), (5.8) on the $\mathbf{B}, \mathbf{L}$ terms and (5.9) on the $\mathbf{W}$ terms, we conclude that

$$
U_{k} \leq C^{k} U_{k-1}^{\min \left\{\frac{5}{3}-\frac{2}{3 p_{3}}, \frac{4}{3}\right\}}
$$

provided $U_{k-1}<1$. Here $p_{3}>1$ ensures the index is strictly greater than 1 . Since

$$
\begin{aligned}
U_{0} & =\sup _{t \in(-1,0)} \int\left|v_{0}\right|^{2} \mathrm{~d} x+\int_{-1}^{0} \int_{B_{1}} d_{0}^{2} \mathrm{~d} x \mathrm{~d} t \\
& =\sup _{t \in(-1,0)} \int|v|^{2} \mathrm{~d} x+\int_{-1}^{0} \int_{B_{1}}|\nabla v|^{2} \mathrm{~d} x \mathrm{~d} t \leq \eta
\end{aligned}
$$

by Proposition 4.1, we know that if $\eta$ is small enough, $U_{k} \rightarrow 0$ as $k \rightarrow \infty$. So in $Q_{\frac{1}{2}},|v| \leq 1$ a.e.. This finishes the proof of Proposition 5.1

\section{Local Study: Part Three, More Regularity}

In this section, we will show that the vorticity $\omega$ is smooth in space. We will only work with the vorticity equation from now on. After the previous two steps, in $B_{\frac{1}{2}}$ we should always decompose $u=v+w$, because $v$ is bounded and $w$ is harmonic.

For convenience, given a vector $\omega$, we denote

$$
\omega^{0}:=\frac{\omega}{|\omega|}, \quad \omega^{\alpha}:=|\omega|^{\alpha} \omega^{0}, \alpha \in \mathbb{R} .
$$


Let $\partial$. be the partial derivative in any space direction or time, then we have

$$
\begin{aligned}
\partial_{\bullet}\left(|\omega|^{\alpha}\right) & =\alpha \omega^{\alpha-1} \cdot \partial_{\bullet} \omega \\
\partial_{\bullet}\left(\omega^{\alpha}\right) & =|\omega|^{\alpha-1} \partial_{\bullet} \omega+(\alpha-1)\left(\omega^{\alpha-2} \cdot \partial_{\bullet} \omega\right) \omega \\
\frac{1}{\alpha} \partial_{\bullet} \partial_{\bullet}\left(|\omega|^{\alpha}\right) & =|\omega|^{\alpha-2}\left|\partial_{\bullet} \omega\right|^{2}+(\alpha-2)\left(\omega^{\frac{\alpha}{2}-1} \cdot \partial_{\bullet} \omega\right)^{2}+\omega^{\alpha-1} \cdot \partial_{\bullet} \partial_{\bullet} \omega \\
& \geq(\alpha-1)\left(\omega^{\frac{\alpha}{2}-1} \cdot \partial_{\bullet} \omega\right)^{2}+\omega^{\alpha-1} \cdot \partial_{\bullet} \partial_{\bullet} \omega \\
& =\frac{4(\alpha-1)}{\alpha^{2}}\left|\partial_{\bullet} \omega^{\frac{\alpha}{2}}\right|^{2}+\omega^{\alpha-1} \cdot \partial_{\bullet} \partial_{\bullet} \omega .
\end{aligned}
$$

6.1. Bound Vorticity in the Energy Space. We will first show $\omega$ is bounded in the energy space.

Proposition 6.1. If $u=v+w$ in $Q_{\frac{1}{2}}$, where $v, w$ are bounded in

$$
\begin{aligned}
\|v\|_{L^{\infty}\left(Q_{\frac{1}{2}}\right)}+\|\nabla v\|_{L^{2}\left(Q_{\frac{1}{2}}\right)} & \leq 2, \\
\|\operatorname{curl} w\|_{L_{t}^{2} L_{x}^{\frac{3}{2}}\left(Q_{\frac{1}{2}}\right)}+\|w\|_{L_{t}^{\frac{4}{3}} \operatorname{Lip}_{x}\left(Q_{\frac{1}{2}}\right)} & \leq 2,
\end{aligned}
$$

$\omega=\operatorname{curl} u$ solves the vorticity equation (1.6), then

(a) $\left\|\omega^{\frac{3}{4}}\right\|_{\mathcal{E}\left(Q_{\frac{1}{4}}\right)} \leq C$

(b) $\|\omega\|_{\mathcal{E}\left(Q_{\frac{1}{8}}\right)} \leq C$,

Proof of Proposition 6.1 (a). We fix a pair of smooth space-time cut-off functions $\varrho$ and $\varsigma$ which satisfy

$$
\mathbf{1}_{Q_{\frac{1}{8}}} \leq \varsigma \leq \mathbf{1}_{Q_{\frac{1}{4}}} \leq \varrho \leq \mathbf{1}_{Q_{\frac{1}{2}}} .
$$

Take the dot product of the vorticity equation (1.6) with $\frac{3}{2} \omega^{\frac{1}{2}}$ :

$$
\begin{aligned}
\frac{3}{2} \omega^{\frac{1}{2}} \cdot \partial_{t} \omega & =\partial_{t}\left(|\omega|^{\frac{3}{2}}\right), \\
\frac{3}{2} \omega^{\frac{1}{2}} \cdot(u \cdot \nabla) \omega & =(u \cdot \nabla)\left(|\omega|^{\frac{3}{2}}\right), \\
\frac{3}{2} \omega^{\frac{1}{2}} \cdot \Delta \omega & \leq \Delta\left(|\omega|^{\frac{3}{2}}\right)-\frac{4}{3}\left|\nabla \omega^{\frac{3}{4}}\right|^{2} .
\end{aligned}
$$

Therefore,

$$
\left(\partial_{t}+u \cdot \nabla-\Delta\right)\left(|\omega|^{\frac{3}{2}}\right)+\frac{3}{2} \omega \cdot \nabla u \cdot \omega^{\frac{1}{2}}+\frac{4}{3}\left|\nabla \omega^{\frac{3}{4}}\right|^{2} \leq 0 .
$$

Multiply by $\varrho^{6}$ then integrate over space,

$$
\int \varrho^{6}\left(\partial_{t}+u \cdot \nabla-\Delta\right)\left(|\omega|^{\frac{3}{2}}\right) \mathrm{d} x+\frac{4}{3} \int \varrho^{6}\left|\nabla \omega^{\frac{3}{4}}\right|^{2} \mathrm{~d} x \leq-\frac{3}{2} \int \varrho^{6} \omega \cdot \nabla u \cdot \omega^{\frac{1}{2}} \mathrm{~d} x .
$$

For the left hand side, we can integrate by part,

$$
\begin{aligned}
& \int \varrho^{6}\left(\partial_{t}+u \cdot \nabla-\Delta\right)\left(|\omega|^{\frac{3}{2}}\right) \mathrm{d} x \\
& \quad=\frac{\mathrm{d}}{\mathrm{d} t} \int \varrho^{6}|\omega|^{\frac{3}{2}} \mathrm{~d} x-\int\left(\left(\partial_{t}+u \cdot \nabla+\Delta\right) \varrho^{6}\right)|\omega|^{\frac{3}{2}} \mathrm{~d} x,
\end{aligned}
$$


where the latter can be controlled by

$$
\int\left(\left(\partial_{t}+u \cdot \nabla+\Delta\right) \varrho^{6}\right)|\omega|^{\frac{3}{2}} \mathrm{~d} x \leq C\left(1+\|u\|_{L^{\infty}\left(B_{\frac{1}{2}}\right)}\right) \int \varrho^{4}|\omega|^{\frac{3}{2}} \mathrm{~d} x .
$$

For the right hand side, using $u=v+w$ over the support of $\varrho$ we can separate

$$
\int \varrho^{6} \omega \cdot \nabla u \cdot \omega^{\frac{1}{2}} \mathrm{~d} x=\int \varrho^{6} \omega \cdot \nabla v \cdot \omega^{\frac{1}{2}} \mathrm{~d} x+\int \varrho^{6} \omega \cdot \nabla w \cdot \omega^{\frac{1}{2}} \mathrm{~d} x,
$$

The $\nabla v$ term can be controlled by

$$
\begin{aligned}
\int \varrho^{6} \omega \cdot \nabla v \cdot \omega^{\frac{1}{2}} \mathrm{~d} x & =-\int \omega \cdot \nabla\left(\varrho^{6} \omega^{\frac{1}{2}}\right) \cdot v \mathrm{~d} x \\
& =-\int \varrho^{6} \omega \cdot \nabla\left(\omega^{\frac{1}{2}}\right) \cdot v \mathrm{~d} x-\int \omega \cdot\left(\omega^{\frac{1}{2}} \otimes \nabla \varrho^{6}\right) \cdot v \mathrm{~d} x,
\end{aligned}
$$

where

$$
\begin{aligned}
\omega \cdot \nabla\left(\omega^{\frac{1}{2}}\right) & =|\omega|^{-\frac{1}{2}} \omega \cdot \nabla \omega-\frac{1}{2}\left(\omega \cdot \nabla \omega \cdot \omega^{-\frac{3}{2}}\right) \omega=\omega^{\frac{1}{2}} \cdot \nabla \omega-\frac{1}{2}\left(\omega^{\frac{1}{2}} \cdot \nabla \omega \cdot \omega^{0}\right) \omega^{0} \\
\Rightarrow\left|\omega \cdot \nabla\left(\omega^{\frac{1}{2}}\right)\right| & \leq\left|\frac{3}{2} \omega^{\frac{1}{2}} \cdot \nabla \omega\right|=2|\omega|^{\frac{3}{4}}\left|\frac{3}{4} \omega^{-\frac{1}{4}} \cdot \nabla \omega\right|=\left.2|\omega|^{\frac{3}{4}}|\nabla| \omega\right|^{\frac{3}{4}} \mid \\
& \leq 2|\omega|^{\frac{3}{4}}\left|\nabla \omega^{\frac{3}{4}}\right| \leq|\omega|^{\frac{3}{2}}+\left|\nabla \omega^{\frac{4}{3}}\right|^{2} .
\end{aligned}
$$

Here the second to the last inequality is due to $\partial_{i}|\omega|^{\frac{3}{4}}=\partial_{i} \omega^{\frac{3}{4}} \cdot \omega^{0}$. Since $|v| \leq 1$ over the support of $\varrho$,

$$
\int \varrho^{6} \omega \cdot \nabla\left(\omega^{\frac{1}{2}}\right) \cdot v \mathrm{~d} x \leq \int \varrho^{6}|\omega|^{\frac{3}{2}} \mathrm{~d} x+\int \varrho^{6}\left|\nabla \omega^{\frac{3}{4}}\right|^{2} \mathrm{~d} x .
$$

By using (6.4)-(6.8) in (6.3), we conclude

$$
\begin{aligned}
& \frac{\mathrm{d}}{\mathrm{d} t} \int \varrho^{6}|\omega|^{\frac{3}{2}} \mathrm{~d} x+\frac{4}{3} \int \varrho^{6}\left|\nabla \omega^{\frac{3}{4}}\right|^{2} \mathrm{~d} x \\
& \leq \int\left[\left(\partial_{t}+u \cdot \nabla+\Delta\right) \varrho^{6}\right]|\omega|^{\frac{3}{2}} \mathrm{~d} x \\
& \quad+\int \varrho^{6} \omega \cdot \nabla w \cdot \omega^{\frac{1}{2}} \mathrm{~d} x \\
& \quad+\int \omega \cdot\left(\omega^{\frac{1}{2}} \otimes \nabla \varrho^{6}\right) \cdot v \mathrm{~d} x \\
& \quad+\int \varrho^{6}|\omega|^{\frac{3}{2}} \mathrm{~d} x+\int \varrho^{6}\left|\nabla \omega^{\frac{3}{4}}\right|^{2} \mathrm{~d} x \\
& \frac{\mathrm{d}}{\mathrm{d} t} \int \varrho^{6}|\omega|^{\frac{3}{2}} \mathrm{~d} x+\frac{1}{3} \int \varrho^{6}\left|\nabla \omega^{\frac{3}{4}}\right|^{2} \mathrm{~d} x \\
& \leq C\left(1+\|u(t)\|_{L^{\infty}\left(B_{\frac{1}{2}}\right)}+\|\nabla w(t)\|_{L^{\infty}\left(B_{\frac{1}{2}}\right)}\right) \int \varrho^{4}|\omega|^{\frac{3}{2}} \mathrm{~d} x .
\end{aligned}
$$

By Hölder's inequality,

$$
\int \varrho^{4}|\omega|^{\frac{3}{2}} \mathrm{~d} x \leq\|\omega(t)\|_{L^{\frac{3}{2}\left(B_{\frac{1}{2}}\right)}}^{\frac{1}{2}}\left(\int \varrho^{6}|\omega|^{\frac{3}{2}} \mathrm{~d} x\right)^{\frac{2}{3}} .
$$

Therefore we can write

$$
\frac{\mathrm{d}}{\mathrm{d} t} \int \varrho^{6}|\omega|^{\frac{3}{2}} \mathrm{~d} x+\frac{1}{3} \int \varrho^{6}\left|\nabla \omega^{\frac{3}{4}}\right|^{2} \mathrm{~d} x \leq C \Phi(t)\left(1+\int \varrho^{6}|\omega|^{\frac{3}{2}} \mathrm{~d} x\right),
$$


where

$$
\begin{aligned}
\Phi(t)= & \left(1+\|u(t)\|_{L^{\infty}\left(B_{\frac{1}{2}}\right)}+\|\nabla w(t)\|_{L^{\infty}\left(B_{\frac{1}{2}}\right)}\right)\|\omega(t)\|_{L^{\frac{3}{2}}\left(B_{\frac{1}{2}}\right)}^{\frac{1}{2}} \\
\leq & \left(2+\|w(t)\|_{L^{\infty}\left(B_{\frac{1}{2}}\right)}+\|\nabla w(t)\|_{L^{\infty}\left(B_{\frac{1}{2}}\right)}\right) \\
& \times\left(\|\operatorname{curl} v(t)\|_{L^{\frac{3}{2}\left(B_{\frac{1}{2}}\right)}}^{\frac{1}{2}}+\|\operatorname{curl} w(t)\|_{L^{\frac{3}{2}\left(B_{\frac{1}{2}}\right)}}^{\frac{1}{2}}\right)
\end{aligned}
$$

since $u=w+v$, and $|v| \leq 1$ inside $B_{\frac{1}{2}}$. By (6.1),

$$
\int_{-\frac{1}{4}}^{0} \Phi(t) \mathrm{d} t \lesssim\left(1+\|w\|_{L_{t}^{\frac{4}{3}} \operatorname{Lip}_{x}\left(Q_{\frac{1}{2}}\right)}\right)\left(\|\nabla v\|_{L^{2}\left(Q_{\frac{1}{2}}\right)}^{\frac{1}{2}}+\|\operatorname{curl} w(t)\|_{L_{t}^{2} L_{x}^{\frac{3}{2}}\left(Q_{\frac{1}{2}}\right)}^{\frac{1}{2}}\right) \leq C .
$$

So by Grönwall's inequality,

$$
\left\|\omega^{\frac{3}{4}}\right\|_{L_{t}^{\infty} L_{x}^{2} \cap L_{t}^{2} \dot{H}_{x}^{1}\left(Q_{\frac{1}{4}}\right)}^{2} \leq e^{C}-1
$$

Proof of Proposition 6.1 (b). From Proposition 6.1 (a) and Sobolev embedding,

$$
\|\omega\|_{L_{t}^{\infty} L_{x}^{\frac{3}{2}} \cap L_{t}^{\frac{3}{2}} L_{x}^{\frac{9}{2}}\left(Q_{\frac{1}{4}}\right)} \leq C
$$

this interpolates the space

$$
\|\omega\|_{L_{t}^{4} L_{x}^{2}\left(Q_{\frac{1}{4}}\right)} \leq C .
$$

Multiply the vorticity equation (1.6) by $\varsigma^{2} \omega$ then integrate over $\mathbb{R}^{3}$,

$$
\begin{aligned}
\frac{\mathrm{d}}{\mathrm{d} t} \int \varsigma^{2} \frac{|\omega|^{2}}{2} \mathrm{~d} x+\int \varsigma^{2}|\nabla \omega|^{2} \mathrm{~d} x= & \left(\partial_{t} \varsigma^{2}+\Delta \varsigma^{2}\right) \frac{|\omega|^{2}}{2} \mathrm{~d} x \\
& -\int(u \cdot \nabla \omega) \cdot \varsigma^{2} \omega \mathrm{d} x \\
& +\int(\omega \cdot \nabla u) \cdot \varsigma^{2} \omega \mathrm{d} x .
\end{aligned}
$$

The first integral is $L^{1}$ in time because $\omega \in L_{t}^{4} L_{x}^{2}$. For the second,

$$
\begin{aligned}
\int(u \cdot \nabla \omega) \cdot \varsigma^{2} \omega \mathrm{d} x & =\int \varsigma^{2} u \cdot \nabla \frac{|\omega|^{2}}{2} \mathrm{~d} x \\
& =-\int \frac{|\omega|^{2}}{2} u \cdot \nabla \varsigma^{2} \mathrm{~d} x \\
& =-\int \varsigma|\omega|^{2} u \cdot \nabla \varsigma \\
& \leq\|\varsigma \omega\|_{L^{2}}\|u \cdot \nabla \varsigma|\omega|\|_{L^{2}},
\end{aligned}
$$

the latter is bounded $L^{1}$ in time, by $u \in L_{t}^{\frac{4}{3}} L_{x}^{\infty}$ and $\omega \in L_{t}^{4} L_{x}^{2}$. For the third integral,

$$
\int(\omega \cdot \nabla u) \cdot \varsigma^{2} \omega \mathrm{d} x=\int(\omega \cdot \nabla v) \cdot \varsigma^{2} \omega \mathrm{d} x+\int(\omega \cdot \nabla w) \cdot \varsigma^{2} \omega \mathrm{d} x .
$$


$w$ is bounded in $L_{t}^{\frac{4}{3}} \operatorname{Lip}_{x}$, and for $v$,

$$
\begin{aligned}
\int(\omega \cdot \nabla v) \cdot \varsigma^{2} \omega \mathrm{d} x & =\int v \cdot(\omega \cdot \nabla)\left(\varsigma^{2} \omega\right) \mathrm{d} x \\
& =\int v \cdot \omega\left(\omega \cdot \nabla \varsigma^{2}\right) \mathrm{d} x+\int v \cdot\left(\varsigma^{2} \omega \cdot \nabla \omega\right) \mathrm{d} x .
\end{aligned}
$$

The former is $L^{1}$ in time, while the latter can be bounded by Cauchy-Schwartz,

$$
\int v \cdot\left(\varsigma^{2} \omega \cdot \nabla \omega\right) \mathrm{d} x \leq \frac{1}{2} \int|v \otimes \varsigma \omega|^{2} \mathrm{~d} x+\frac{1}{2} \int \varsigma^{2}|\nabla \omega|^{2} \mathrm{~d} x .
$$

In conclusion,

$$
\begin{aligned}
& \frac{\mathrm{d}}{\mathrm{d} t} \int \varsigma^{2} \frac{|\omega|^{2}}{2} \mathrm{~d} x+\frac{1}{2} \int \varsigma^{2}|\nabla \omega|^{2} \mathrm{~d} x \\
& \leq C\|\omega(t)\|_{L^{2}\left(B_{\frac{1}{4}}\right)}^{2}+C\|u(t)\|_{L^{\infty}\left(B_{\frac{1}{4}}\right)}\|\omega(t)\|_{L^{2}\left(B_{\frac{1}{4}}\right)}\|\varsigma \omega(t)\|_{L^{2}} \\
& \quad+C\|\nabla w\|_{L^{\infty}\left(B_{\frac{1}{4}}\right)}\|\varsigma \omega(t)\|_{L^{2}}^{2} \\
& \leq C \Phi(t)\left(1+\int \varsigma^{2} \frac{|\omega|^{2}}{2} \mathrm{~d} x\right)
\end{aligned}
$$

where

$$
\Phi(t)=\|\omega(t)\|_{L^{2}\left(B_{\frac{1}{4}}\right)}^{2}+\|u(t)\|_{L^{\infty}\left(B_{\frac{1}{4}}\right)}\|\omega(t)\|_{L^{2}\left(B_{\frac{1}{4}}\right)}+\|\nabla w(t)\|_{L^{\infty}\left(B_{\frac{1}{4}}\right)},
$$

whose integral is bounded using (6.1),

$$
\int_{-\frac{1}{16}}^{0} \Phi(t) \mathrm{d} t \leq\|\omega\|_{L^{2}\left(Q_{\frac{1}{4}}\right)}^{2}+\|u\|_{L_{t}^{\frac{4}{3}} L_{x}^{\infty}\left(Q_{\frac{1}{4}}\right)}\|\omega\|_{L_{t}^{4} L_{x}^{2}\left(Q_{\frac{1}{4}}\right)}+\|\nabla w\|_{L_{t}^{\frac{4}{3}} L_{x}^{\infty}\left(Q_{\frac{1}{4}}\right)} \leq C .
$$

By a Grönwall argument, we have

$$
\|\omega\|_{L_{t}^{\infty} L_{x}^{2} \cap L_{t}^{2} \dot{H}_{x}^{1}\left(Q_{\frac{1}{8}}\right)}^{2} \leq e^{C}-1 .
$$

6.2. Bound Higher Derivatives in the Energy Space. Now we iteratively show higher derivatives of vorticity by induction.

Proposition 6.2. For any $n \geq 1$, if $u=v+w$ in $Q_{8^{-n}}$, where $v, w$ are bounded in

$$
\begin{aligned}
\|v\|_{L^{\infty}\left(Q_{8^{-n} / 2}\right)}+\|v\|_{L_{t}^{2} H_{x}^{n+1}\left(Q_{8^{-n} / 2}\right)} & \leq c_{n}, \\
\|w\|_{L_{t}^{\frac{4}{3}} C_{x}^{n+1}\left(Q_{8^{-n} / 2}\right)} & \leq c_{n},
\end{aligned}
$$

for some constant $c_{n}, \omega=\operatorname{curl} u$ solves the vorticity equation (1.6), and is bounded in

$$
\|\omega\|_{L_{t}^{\infty} H_{x}^{n-1} \cap L_{t}^{2} H_{x}^{n}\left(Q_{8-n / 2}\right)} \leq c_{n},
$$

then for any multiindex $\alpha$ with $|\alpha|=n$,

(a) $\left\|\nabla^{\alpha} \omega^{\frac{3}{4}}\right\|_{\mathcal{E}\left(Q_{8^{-n} / 4}\right)} \leq C_{n}$

(b) $\left\|\nabla^{\alpha} \omega\right\|_{\mathcal{E}\left(Q_{8-n-1}\right)} \leq C_{n}$

for some $C_{n}$ depending on $c_{n}$ and $n$. 
Proof of Proposition 6.2 (a). Similarly we fix smooth cut-off functions $\varrho_{n}$ and $\varsigma_{n}$ which satisfy

$$
\mathbf{1}_{Q_{8^{-n-1}}} \leq \varsigma_{n} \leq \mathbf{1}_{Q_{8^{-n} / 4}} \leq \varrho_{n} \leq \mathbf{1}_{Q_{8^{-n} / 2}} .
$$

Differentiate (1.6) by $\nabla^{\alpha}$,

$$
\partial_{t} \nabla^{\alpha} \omega+u \cdot \nabla \nabla^{\alpha} \omega-\nabla^{\alpha} \omega \cdot \nabla u+\mathbf{P}_{\alpha}=\Delta \nabla^{\alpha} \omega
$$

where

$$
\mathbf{P}_{\alpha}=\sum_{\beta<\alpha}\left(\begin{array}{l}
\alpha \\
\beta
\end{array}\right) \operatorname{curl}\left(\nabla^{\beta} \omega \times \nabla^{\alpha-\beta} u\right) .
$$

Multiply (6.12) by $\frac{3}{2} \varrho_{n}^{6}\left(\nabla^{\alpha} \omega\right)^{\frac{1}{2}}$ then integrate in space,

$$
\begin{aligned}
& \frac{\mathrm{d}}{\mathrm{d} t} \int \varrho_{n}^{6}\left|\nabla^{\alpha} \omega\right|^{\frac{3}{2}} \mathrm{~d} x+\frac{4}{3} \int \varrho_{n}^{6}\left|\nabla \nabla^{\alpha} \omega^{\frac{3}{4}}\right|^{2} \mathrm{~d} x \\
& \leq \int\left[\left(\partial_{t}+u \cdot \nabla+\Delta\right) \varrho_{n}^{6}\right]\left|\nabla^{\alpha} \omega\right|^{\frac{3}{2}} \mathrm{~d} x \\
& +\int \varrho_{n}^{6} \nabla^{\alpha} \omega \cdot \nabla w \cdot\left(\nabla^{\alpha} \omega\right)^{\frac{1}{2}} \mathrm{~d} x \\
& +\int \nabla^{\alpha} \omega \cdot\left(\left(\nabla^{\alpha} \omega\right)^{\frac{1}{2}} \otimes \nabla \varrho_{n}^{6}\right) \cdot v \mathrm{~d} x \\
& +\|v\|_{L^{\infty}\left(Q_{8^{-n}}\right)}^{2} \int \varrho_{n}^{6}\left|\nabla^{\alpha} \omega\right|^{\frac{3}{2}} \mathrm{~d} x+\int \varrho_{n}^{6}\left|\nabla \nabla^{\alpha} \omega^{\frac{3}{4}}\right|^{2} \mathrm{~d} x \\
& +\frac{3}{2} \int \varrho_{n}^{6}\left(\nabla^{\alpha} \omega\right)^{\frac{1}{2}} \cdot \mathbf{P}_{\alpha} \mathrm{d} x
\end{aligned}
$$

same as in the proof of Proposition 6.1 (a). So

$$
\begin{aligned}
& \frac{\mathrm{d}}{\mathrm{d} t} \int \varrho_{n}^{6}\left|\nabla^{\alpha} \omega\right|^{\frac{3}{2}} \mathrm{~d} x+\frac{1}{3} \int \varrho_{n}^{6}\left|\nabla \nabla^{\alpha} \omega^{\frac{3}{4}}\right|^{2} \mathrm{~d} x \\
& \leq C \\
& \quad\left(1+\|u(t)\|_{L^{\infty}\left(B_{8-n}\right)}+\|\nabla w(t)\|_{L^{\infty}\left(B_{8-n}\right)}\right) \int \varrho_{n}^{4}\left|\nabla^{\alpha} \omega\right|^{\frac{3}{2}} \mathrm{~d} x \\
& \quad+\frac{3}{2} \int \varrho_{n}^{6}\left(\nabla^{\alpha} \omega\right)^{\frac{1}{2}} \cdot \mathbf{P}_{\alpha} \mathrm{d} x .
\end{aligned}
$$

Terms other than $\mathbf{P}_{\alpha}$ are dealt with by the same way as in Proposition 6.1.

$$
\int \varrho_{n}^{4}\left|\nabla^{\alpha} \omega\right|^{\frac{3}{2}} \mathrm{~d} x \leq \|\left.\nabla^{\alpha} \omega(t)\right|_{L^{\frac{3}{2}}\left(B_{8^{-n}}\right)} ^{\frac{1}{2}}\left(\int \varrho_{n}^{6}\left|\nabla^{\alpha} \omega\right|^{\frac{3}{2}} \mathrm{~d} x\right)^{\frac{2}{3}} .
$$

The induction condition (6.11) ensures that $\left\|\nabla^{\alpha} \omega\right\|_{L^{2}\left(Q_{8-n}\right)} \leq c_{n}$. Therefore

$$
\begin{gathered}
\int_{-8^{-2 n}}^{0}\left(1+\|u(t)\|_{L^{\infty}\left(B_{8^{-n}}\right)}+\|\nabla w(t)\|_{L^{\infty}\left(B_{8^{-n}}\right)}\right)\left\|\nabla^{\alpha} \omega(t)\right\|_{L^{\frac{3}{2}}\left(B_{8^{-n}}\right)}^{\frac{1}{2}} \mathrm{~d} t \\
\lesssim\left(1+\|v\|_{L^{\infty}\left(B_{8^{-n}}\right)}+\|w\|_{L_{t}^{\frac{4}{3}} C_{x}^{1}\left(B_{8^{-n}}\right)}\right)\left\|\nabla^{\alpha} \omega\right\|_{L^{2}\left(Q_{8^{-n}}\right)}^{\frac{1}{2}} \leq C_{n} .
\end{gathered}
$$

Now let's focus on $\mathbf{P}_{\alpha}$.

$$
\left|\mathbf{P}_{\alpha}\right| \lesssim \sum_{k=0}^{n}\left|\nabla^{k} \omega\right|\left|\nabla^{n-k+1} u\right| \leq \sum_{k=0}^{n}\left|\nabla^{k} \omega\right|\left|\nabla^{n-k+1} v\right|+\sum_{k=0}^{n}\left|\nabla^{k} \omega\right|\left|\nabla^{n-k+1} w\right| .
$$


We denote

$$
\mathbf{P}_{v, k}=\left|\nabla^{k} \omega\right|\left|\nabla^{n-k+1} v\right|, \quad \mathbf{P}_{w, k}=\left|\nabla^{k} \omega\right|\left|\nabla^{n-k+1} w\right| .
$$

First we estimate $\mathbf{P}_{v, k}$. By (6.9) and (6.11), when $k=0$,

$$
\left\|\mathbf{P}_{v, 0}\right\|_{L_{t}^{1} L_{x}^{\frac{3}{2}}\left(Q_{8^{-n}}\right)} \leq\|\omega\|_{L_{t}^{2} L_{x}^{6}\left(Q_{8^{-n}}\right)}\left\|\nabla^{n+1} v\right\|_{L_{t}^{2} L_{x}^{2}\left(Q_{8^{-n}}\right)} \leq C_{n}
$$

and when $0<k \leq n$,

$$
\left\|\mathbf{P}_{v, k}\right\|_{L_{t}^{1} L_{x}^{\frac{3}{2}}\left(Q_{8^{-n}}\right)} \leq\left\|\nabla^{k} \omega\right\|_{L_{t}^{2} L_{x}^{2}\left(Q_{8^{-n}}\right)}\left\|\nabla^{n+1-k} v\right\|_{L_{t}^{2} L_{x}^{6}\left(Q_{8^{-n}}\right)} \leq C_{n} .
$$

Next we estimate $\mathbf{P}_{w, k}$. When $0 \leq k<n$,

$$
\left\|\mathbf{P}_{w, k}\right\|_{L_{t}^{1} L_{x}^{\frac{3}{2}}\left(Q_{8^{-n}}\right)} \leq\left\|\nabla^{k} \omega\right\|_{L_{t}^{\infty} L_{x}^{2}\left(Q_{8^{-n}}\right)}\left\|\nabla^{n+1-k} w\right\|_{L_{t}^{\frac{4}{3}} L_{x}^{\infty}\left(Q_{8^{-n}}\right)} \leq C_{n} .
$$

Finally, when $k=n$,

$$
\left|\mathbf{P}_{w, n}\right|_{L_{x}^{\frac{3}{2}}\left(B_{8^{-n}}\right)} \leq\left|\nabla^{n} \omega\right||\nabla w|
$$

Therefore,

$$
\begin{aligned}
& \int \varrho_{n}^{6}\left(\nabla^{\alpha} \omega\right)^{\frac{1}{2}} \cdot \mathbf{P}_{\alpha} \mathrm{d} x \leq\left(1+\int \varrho_{n}^{6}\left|\nabla^{\alpha} \omega\right|^{\frac{3}{2}} \mathrm{~d} x\right) \\
& \quad \times\left(\sum_{k=0}^{n}\left\|\mathbf{P}_{v, k}\right\|_{L_{x}^{\frac{3}{2}}\left(B_{8^{-n}}\right)}+\sum_{k=0}^{n-1}\left\|\mathbf{P}_{w, k}\right\|_{L_{x}^{\frac{3}{2}}\left(B_{8^{-n}}\right)}+\|\nabla w\|_{L_{x}^{\infty}\left(B_{8^{-n}}\right)}\right)
\end{aligned}
$$

In conclusion, we have shown that

$$
\frac{\mathrm{d}}{\mathrm{d} t} \int \varrho_{n}^{6}\left|\nabla^{\alpha} \omega\right|^{\frac{3}{2}} \mathrm{~d} x+\frac{1}{3} \int \varrho_{n}^{6}\left|\nabla \nabla^{\alpha} \omega^{\frac{3}{4}}\right|^{2} \mathrm{~d} x \leq C \Phi(t)\left(1+\int \varrho_{n}^{6}\left|\nabla^{n} \omega\right|^{\frac{3}{2}} \mathrm{~d} x\right),
$$

where

$$
\begin{aligned}
\Phi(t)=(1 & \left.+\|u(t)\|_{L^{\infty}\left(B_{8-n}\right)}+\|\nabla w(t)\|_{L^{\infty}\left(B_{8^{-n}}\right)}\right)\left\|\nabla^{\alpha} \omega(t)\right\|_{L^{\frac{3}{2}}\left(B_{8^{-n}}\right)}^{\frac{1}{2}} \\
& +\sum_{k=0}^{n}\left\|\mathbf{P}_{v, k}\right\|_{L_{x}^{\frac{3}{2}}\left(B_{8^{-n}}\right)}+\sum_{k=0}^{n-1}\left\|\mathbf{P}_{w, k}\right\|_{L_{x}^{\frac{3}{2}}\left(B_{8^{-n}}\right)}+\|\nabla w\|_{L_{x}^{\infty}\left(B_{8^{-n}}\right)}
\end{aligned}
$$

with integral

$$
\int_{-8^{-2 n} / 4}^{0} \Phi(t) \mathrm{d} t \leq C_{n}
$$

Taking the sum over all multi-index $\alpha$ with size $|\alpha|=n$, we have

$$
\frac{\mathrm{d}}{\mathrm{d} t} \int \varrho_{n}^{6}\left|\nabla^{n} \omega\right|^{\frac{3}{2}} \mathrm{~d} x+\frac{1}{3} \int \varrho_{n}^{6}\left|\nabla^{n+1} \omega^{\frac{3}{4}}\right|^{2} \mathrm{~d} x \leq C \Phi(t)\left(1+\int \varrho_{n}^{6}\left|\nabla^{n+1} \omega\right|^{\frac{3}{2}} \mathrm{~d} x\right),
$$

Finally, Grönwall inequality gives

$$
\left\|\left|\nabla^{n+1} \omega\right|^{\frac{3}{4}}\right\|_{L_{t}^{\infty} L_{x}^{2} \cap L_{t}^{2} \dot{H}_{x}^{1}\left(Q_{8-n / 4}\right)} \leq C_{n} .
$$


Proof of Proposition 6.2 (b). Now we multiply (6.12) by $\varsigma_{n}^{2} \nabla^{\alpha} \omega$ then integrate over $\mathbb{R}^{3}$

$$
\begin{aligned}
\frac{\mathrm{d}}{\mathrm{d} t} \int \varsigma_{n}^{2} \frac{\left|\nabla^{\alpha} \omega\right|^{2}}{2} \mathrm{~d} x+\int \varsigma_{n}^{2}\left|\nabla \nabla^{\alpha} \omega\right|^{2} \mathrm{~d} x= & \int\left(\partial_{t} \varsigma_{n}^{2}+\Delta \varsigma_{n}^{2}\right) \frac{\left|\nabla^{\alpha} \omega\right|^{2}}{2} \mathrm{~d} x \\
& -\int\left(u \cdot \nabla \nabla^{\alpha} \omega\right) \cdot \varsigma_{n}^{2} \nabla^{\alpha} \omega \mathrm{d} x \\
& +\int\left(\nabla^{\alpha} \omega \cdot \nabla u\right) \cdot \varsigma_{n}^{2} \nabla^{\alpha} \omega \mathrm{d} x \\
& +\int \varsigma_{n}^{2} \nabla^{\alpha} \omega \cdot \mathbf{P}_{\alpha} \mathrm{d} x
\end{aligned}
$$

For the same reason, the only term that we need to take care of is $\mathbf{P}_{\alpha}$ term, and the others are dealt the same as in Proposition 6.1 (b):

$$
\begin{gathered}
\int\left(\partial_{t} \varsigma_{n}^{2}+\Delta \varsigma_{n}^{2}\right) \frac{\left|\nabla^{\alpha} \omega\right|^{2}}{2} \mathrm{~d} x-\int\left(u \cdot \nabla \nabla^{\alpha} \omega\right) \cdot \varsigma_{n}^{2} \nabla^{\alpha} \omega \mathrm{d} x+\int\left(\nabla^{\alpha} \omega \cdot \nabla u\right) \cdot \varsigma_{n}^{2} \nabla^{\alpha} \omega \mathrm{d} x \\
\lesssim_{n}\left\|\nabla^{\alpha} \omega\right\|_{L^{2}\left(Q_{8^{-n} / 4}\right)}^{2}+\|u\|_{L^{\infty}\left(Q_{8^{-n / 4}}\right)}\left\|\nabla^{\alpha} \omega\right\|_{L^{2}\left(Q_{8^{-n} / 4}\right)}\left(\int \varsigma_{n}^{2} \frac{\left|\nabla^{\alpha} \omega\right|^{2}}{2} \mathrm{~d} x\right)^{\frac{1}{2}} \\
+\|\nabla w\|_{L^{\infty}\left(Q_{8^{-n} / 4}\right)} \int \varsigma_{n}^{2} \frac{\left|\nabla^{\alpha} \omega\right|^{2}}{2} \mathrm{~d} x+\|v\|_{L^{\infty}\left(Q_{8^{-n} / 4}\right)}\left\|\nabla^{\alpha} \omega\right\|_{L^{2}\left(Q_{8^{-n} / 4}\right)}^{2} \\
+\frac{1}{\varepsilon}\|v\|_{L^{\infty}\left(Q_{8^{-n} / 4}\right)}^{2} \int \varsigma_{n}^{2} \frac{\left|\nabla^{\alpha} \omega\right|^{2}}{2} \mathrm{~d} x+\varepsilon \int \varsigma_{n}^{2}\left|\nabla \nabla^{\alpha} \omega\right|^{2} \mathrm{~d} x .
\end{gathered}
$$

The last term can be absorbed into the left, and we will use Grönwall on the remaining terms.

Now we shall focus on the $\mathbf{P}_{\alpha}$ term. From Proposition 6.2 (a), we have

$$
\left\|\nabla^{n} \omega\right\|_{L_{t}^{\infty} L_{x}^{\frac{3}{2}} \cap L_{x}^{\frac{3}{2}} L_{t}^{\frac{9}{2}}\left(Q_{8^{-n / 4}}\right)} \leq C_{n} .
$$

Again by interpolation,

$$
\left\|\nabla^{n} \omega\right\|_{L_{t}^{4} L_{x}^{2}\left(Q_{8-n / 4}\right)} \leq C_{n}, \quad\left\|\nabla^{n} \omega\right\|_{L_{t}^{2} L_{x}^{3}\left(Q_{8^{-n} / 4}\right)} \leq C_{n}
$$

First we estimate $\mathbf{P}_{w, k}$. In this case, for any $0 \leq k \leq n$,

$$
\left\|\mathbf{P}_{w, k}\right\|_{L_{t}^{1} L_{x}^{2}\left(Q_{8^{-n} / 4}\right)} \leq\left\|\nabla^{k} \omega\right\|_{L_{t}^{4} L_{x}^{2}\left(Q_{8^{-n} / 4}\right)}\left\|\nabla^{n+1-k} w\right\|_{L_{t}^{\frac{4}{3} L_{x}^{\infty}\left(Q_{8^{-n}}\right)}} \leq C_{n} .
$$

Then we estimate $\mathbf{P}_{v, k}$. When $0<k \leq n$,

$$
\left\|\mathbf{P}_{v, k}\right\|_{L_{t}^{1} L_{x}^{2}\left(Q_{8-n}\right)} \leq\left\|\nabla^{k} \omega\right\|_{L_{t}^{2} L_{x}^{3}\left(Q_{8-n}\right)}\left\|\nabla^{n+1-k} v\right\|_{L_{t}^{2} L_{x}^{6}\left(Q_{8}-n\right.} \leq C_{n} .
$$

For the case $k=0$ of the $v$ term, we put the curl on $\nabla^{\alpha} \omega$,

$$
\begin{aligned}
\int \varsigma_{n}^{2} & \nabla^{\alpha} \omega \cdot \operatorname{curl}\left(\omega \times \nabla^{\alpha} v\right) \mathrm{d} x \\
& =\int\left(\omega \times \nabla^{\alpha} v\right) \cdot \operatorname{curl}\left(\varsigma_{n}^{2} \nabla^{\alpha} \omega\right) \mathrm{d} x \\
& \leq \int \varsigma_{n}^{2}|\omega|\left|\nabla^{\alpha} v\right|\left|\nabla \nabla^{\alpha} \omega\right|+\varsigma_{n}\left|\nabla \varsigma_{n}\right||\omega|\left|\nabla^{\alpha} v\right|\left|\nabla^{\alpha} \omega\right| \mathrm{d} x \\
& \leq \int \varsigma_{n}^{2}|\omega|^{2}\left|\nabla^{\alpha} v\right|^{2} \mathrm{~d} x+\varepsilon \int \varsigma_{n}^{2}\left|\nabla \nabla^{\alpha} \omega\right|^{2} \mathrm{~d} x+\frac{1}{\varepsilon} \int\left|\nabla \varsigma_{n}\right|^{2}\left|\nabla^{\alpha} \omega\right|^{2} \mathrm{~d} x .
\end{aligned}
$$


where $\left|\nabla \nabla^{\alpha} \omega\right|$ term can be absorbed to the left. By (6.13) and Sobolev embedding,

$$
\|\omega\|_{L_{t}^{\infty} L_{x}^{3}\left(Q_{8-n / 4}\right)} \leq C_{n} .
$$

Therefore

$$
\iint \varsigma_{n}^{2}|\omega|^{2}\left|\nabla^{\alpha} v\right|^{2} \mathrm{~d} x \mathrm{~d} t \leq\|\omega\|_{L_{t}^{\infty} L_{x}^{3}\left(Q_{8^{-n} / 4}\right)}^{2}\left\|\nabla^{\alpha} v\right\|_{L_{t}^{2} L_{x}^{6}\left(Q_{8^{-n} / 4}\right)}^{2} \leq C_{n} .
$$

In conclusion,

$$
\frac{\mathrm{d}}{\mathrm{d} t} \int \varsigma_{n}^{2} \frac{\left|\nabla^{\alpha} \omega\right|^{2}}{2} \mathrm{~d} x+\int \varsigma_{n}^{2}\left|\nabla \nabla^{\alpha} \omega\right|^{2} \mathrm{~d} x \leq C \Phi(t)\left(1+\int \varsigma_{n}^{2} \frac{\left|\nabla^{\alpha} \omega\right|^{2}}{2} \mathrm{~d} x\right),
$$

where

$$
\begin{aligned}
\Phi(t)=\| & \nabla^{\alpha} \omega(t)\left\|_{L^{2}\left(B_{8^{-n} / 4}\right)}^{2}+\right\| u\left\|_{L^{\infty}\left(B_{8^{-n} / 4}\right)}\right\| \nabla^{\alpha} \omega \|_{L^{2}\left(B_{8^{-n} / 4}\right)} \\
& +\|\nabla w\|_{L^{\infty}\left(B_{8^{-n} / 4}\right)}+\|v\|_{L^{\infty}\left(B_{8^{-n} / 4}\right)}\left\|\nabla^{\alpha} \omega\right\|_{L^{2}\left(B_{8^{-n} / 4}\right)}^{2} \\
& +\frac{1}{\varepsilon}\|v\|_{L^{\infty}\left(B_{8^{-n} / 4}\right)}^{2}+\sum_{k=0}^{n}\left\|\mathbf{P}_{w, k}\right\|_{L^{2}\left(B_{8^{-n} / 4}\right)}+\sum_{k=0}^{n-1}\left\|\mathbf{P}_{v, k}\right\|_{L^{2}\left(B_{8^{-n} / 4}\right)} \\
& +\|\omega\|_{L^{3}\left(B_{8^{-n} / 4}\right)}^{2}\left\|\nabla^{\alpha} v\right\|_{L^{6}\left(B_{8^{-n} / 4}\right)}^{2}+\frac{1}{\varepsilon}\left\|\nabla^{\alpha} \omega(t)\right\|_{L^{2}\left(B_{8^{-n} / 4}\right)}^{2}
\end{aligned}
$$

has integral $\int_{-8^{-2 n} / 16}^{0} \Phi(t) \mathrm{d} t \leq C_{n}$. Finally Grönwall inequality gives

$$
\left\|\nabla^{\alpha} \omega\right\|_{L_{t}^{\infty} L_{x}^{2} \cap L_{t}^{2} \dot{H}_{x}^{1}\left(Q_{8-n-1}\right)} \leq C_{n+1} .
$$

\subsection{Proof of the Local Theorem.}

Proof of the Local Theorem 1.3. First, Proposition 4.1 gives

$$
\|v\|_{\mathcal{E}\left(Q_{1}\right)} \leq \eta
$$

where $\eta$ can be chosen arbitrarily small if we pick $\eta_{1}$ small. Next, by Proposition 5.1, we know

$$
\|v\|_{L^{\infty}\left(Q_{\frac{1}{2}}\right)} \leq 1
$$

These two steps implies (6.1). As for (6.2), $\operatorname{curl} w=\varpi$ in $B_{1}$, so we use interpolation in (4.13):

$$
\|\operatorname{curl} w\|_{L_{t}^{2} L_{x}^{\frac{3}{2}}\left(Q_{\frac{1}{2}}\right)} \leq\|\varpi\|_{L_{t}^{2} L_{x}^{\frac{12}{7}}} \leq\|\varpi\|_{L_{t}^{p_{1}} L_{x}^{q_{3}}}^{\frac{1}{2}}\|\varpi\|_{L_{t}^{p_{2}} L_{x}^{q_{4}}}^{\frac{1}{2}} \leq \eta
$$

$w$ is harmonic inside $B_{1}$, therefore

$$
\|w\|_{L_{t}^{\frac{4}{3}} C_{x}^{n}\left(Q_{\frac{1}{2}}\right)} \lesssim_{n}\|w\|_{L_{t}^{\frac{4}{3}} L_{x}^{1}\left(Q_{1}\right)} \leq \eta
$$

due to (4.12) and $p_{1} \geq \frac{4}{3}$. Therefore, we can use Proposition 6.1 to obtain

$$
\|\omega\|_{\mathcal{E}\left(Q_{\frac{1}{8}}\right)} \leq C
$$

The next step is to use Proposition 6.2 iteratively. Suppose for $n \geq 1$ we know that

$$
\left\|\nabla^{n-1} \omega\right\|_{\mathcal{E}\left(Q_{8^{-n}}\right)} \leq c_{n}
$$


which is equivalent to (6.11). Let $\varphi_{n}$ and $\varphi_{n}^{\sharp}$ be a pair of smooth spatial cut-off functions, with

$$
\mathbf{1}_{B_{\frac{1}{8^{n}+4}}} \leq \varphi_{n} \leq \mathbf{1}_{B_{8^{n}+3}}, \quad \mathbf{1}_{B_{\frac{1}{8^{n}+2}}} \leq \varphi_{n}^{\sharp} \leq \mathbf{1}_{B_{\frac{1}{8^{n}+1}}},
$$

and set

$$
v_{n}:=-\operatorname{curl} \varphi_{n}^{\sharp} \Delta^{-1} \varphi_{n} \omega, \quad w_{n}=\varphi_{n} u-v_{n} .
$$

On the one hand, $\nabla v_{n}$ is a Riesz transform of $\varphi_{n} \omega$ up to lower order terms, so by the boundedness of Riesz transform we know

$$
\left\|\nabla^{n+1} v_{n}\right\|_{L^{2}\left(Q_{8^{-n} / 2}\right)} \leq\left\|\nabla^{n} \omega\right\|_{L^{2}\left(Q_{8^{-n}}\right)} \leq c_{n-1} .
$$

On the other hand, we have similar boundedness estimates following Proposition 5.1 as before,

$$
\left\|v_{n}\right\|_{L^{\infty}\left(Q_{8-n / 2}\right)} \leq 1
$$

$w_{n}$ is harmonic in $B_{\frac{1}{8^{n}+4}}$, so we also have

$$
\left\|w_{n}\right\|_{L_{t}^{\frac{4}{3}} C_{x}^{n+1}\left(Q_{8^{-n} / 2}\right)} \lesssim n\left\|w_{n}\right\|_{L_{t}^{\frac{4}{3}} L_{x}^{1}\left(Q_{\frac{1}{8^{n}+4}}\right)} \leq \eta
$$

Therefore, by Proposition 6.2

$$
\left\|\nabla^{n} \omega\right\|_{\mathcal{E}\left(Q_{8^{-n-1}}\right)} \leq C_{n}
$$

By induction, we have

$$
\left\|\nabla^{n} \omega\right\|_{L_{t}^{\infty} L_{x}^{2} \cap L_{t}^{2} \dot{H}_{x}^{1}\left(Q_{8-n-1}\right)} \leq C_{n}
$$

for any $n$. By Sobolev embedding, this implies for any $n$,

$$
\left\|\nabla^{n} \omega\right\|_{L^{\infty}\left(Q_{8^{-n-3}}\right)} \lesssim\left\|\nabla^{n} \omega\right\|_{L_{t}^{\infty} L_{x}^{2}\left(Q_{8^{-n-3}}\right)}+\left\|\nabla^{n+2} \omega\right\|_{L_{t}^{\infty} L_{x}^{2}\left(Q_{8^{-n-3}}\right)} \leq C_{n} .
$$

\section{Appendix A. Suitability of Solutions}

Theorem A.1. Let $u$ be a suitable weak solution to the Navier-Stokes equation in $\mathbb{R}^{3}$. That is, $u \in L_{t}^{\infty} L_{x}^{2} \cap L_{t}^{2} \dot{H}_{x}^{1}$ solves the following equation

$$
\partial_{t} u+u \cdot \nabla u+\nabla P=\Delta u, \quad \operatorname{div} u=0
$$

where $P$ is the pressure, and $u$ satisfies the following local energy inequality,

$$
\partial_{t} \frac{|u|^{2}}{2}+\operatorname{div}\left(u\left(\frac{|u|^{2}}{2}+P\right)\right)+|\nabla u|^{2} \leq \Delta \frac{|u|^{2}}{2} .
$$

Suppose $v \in L_{t}^{\infty} L_{x}^{2} \cap L_{t}^{2} \dot{H}_{x}^{1}$ is compactly supported in space and solves the following equation,

$$
\partial_{t} v+\omega \times v+\nabla \mathbf{R}(u \otimes v)=\Delta v+\mathbf{C}_{v}, \quad \operatorname{div} v=0
$$

where $\omega=\operatorname{curl} u$ is the vorticity, $\mathbf{C}_{v} \in L_{t}^{2} L_{\mathrm{loc}, x}^{\frac{3}{2}}$ is a force term, and

$$
\mathbf{R}=\frac{1}{2} \operatorname{tr}-\Delta^{-1} \operatorname{div} \operatorname{div}
$$

is a symmetric Riesz operator. Moreover, suppose $v$ differs from $\varphi$ u by

$$
\varphi u-v=w \in L_{t}^{\infty} H_{x}^{1} \cap L_{t}^{2} H_{x}^{2}
$$


for some fixed $\varphi \in C_{c}^{\infty}\left(\mathbb{R}^{3}\right)$. Then $v$ satisfies the following local energy inequality,

$$
\partial_{t} \frac{|v|^{2}}{2}+\operatorname{div}(v \mathbf{R}(u \otimes v))+|\nabla v|^{2} \leq \Delta \frac{|v|^{2}}{2}+v \cdot \mathbf{C}_{v} .
$$

Proof. It is well-known that the pressure $P$ can be recovered from $u$ by

$$
P=-\Delta^{-1} \operatorname{div} \operatorname{div}(u \otimes u) .
$$

Since

$$
\begin{aligned}
u \cdot \nabla u+\nabla P & =\nabla \frac{|u|^{2}}{2}+\omega \times u-\nabla \Delta^{-1} \operatorname{div} \operatorname{div}(u \otimes u) \\
& =\omega \times u+\nabla \mathbf{R}(u \otimes u),
\end{aligned}
$$

The Navier-Stokes equation (A.1) can be rewritten as

$$
\partial_{t} u+\omega \times u+\nabla \mathbf{R}(u \otimes u)=\Delta u
$$

and local energy inequality (A.2) can be rewritten as

$$
\partial_{t} \frac{|u|^{2}}{2}+\operatorname{div}(u \mathbf{R}(u \otimes u))+|\nabla u|^{2} \leq \Delta \frac{|u|^{2}}{2},
$$

First, multiply A.5 by $\varphi$,

$$
\partial_{t} \varphi u+\omega \times \varphi u+\nabla \mathbf{R}(u \otimes \varphi u)=\Delta(\varphi u)+[\nabla \mathbf{R}, \varphi](u \otimes u)+[\varphi, \Delta] u .
$$

Denote

$$
\mathbf{C}_{u}=[\nabla \mathbf{R}, \varphi](u \otimes u)+[\varphi, \Delta] u
$$

for these commutator terms. Subtracting the equation of $v$ from this equation of $\varphi u$, we will have the equation for $w$. In summary,

$$
\begin{aligned}
\partial_{t} \varphi u+\omega \times \varphi u+\nabla \mathbf{R}(u \otimes \varphi u) & =\Delta(\varphi u)+\mathbf{C}_{u}, \\
\partial_{t} v+\omega \times v+\nabla \mathbf{R}(u \otimes v) & =\Delta v+\mathbf{C}_{v}, \\
\partial_{t} w+\omega \times w+\nabla \mathbf{R}(u \otimes w) & =\Delta w+\mathbf{C}_{u}-\mathbf{C}_{v} .
\end{aligned}
$$

Recall from Vas10 that $\Delta u \in L_{\operatorname{loc}(t, x)}^{\frac{4}{3}-\varepsilon}$. Since $\Delta w \in L_{t, x}^{2}$, we have $\Delta v \in L_{\operatorname{loc}(t, x)}^{\frac{4}{3}-\varepsilon}$. Therefore, we can multiply (A.7) and (A.8) by $w$, and (A.9) by $\varphi u$ and $v$,

$$
\begin{aligned}
w \cdot \partial_{t}(\varphi u)+w \cdot \omega \times \varphi u+w \cdot \nabla \mathbf{R}(u \otimes \varphi u) & =w \cdot \Delta(\varphi u)+w \cdot \mathbf{C}_{u}, \\
w \cdot \partial_{t} v+w \cdot \omega \times v+w \cdot \nabla \mathbf{R}(u \otimes v) & =w \cdot \Delta v+w \cdot \mathbf{C}_{v} \\
\varphi u \cdot \partial_{t} w+\varphi u \cdot \omega \times w+\varphi u \cdot \nabla \mathbf{R}(u \otimes w) & =\varphi u \cdot \Delta w+\varphi u \cdot\left(\mathbf{C}_{u}-\mathbf{C}_{v}\right) . \\
v \cdot \partial_{t} w+v \cdot \omega \times w+v \cdot \nabla \mathbf{R}(u \otimes w) & =v \cdot \Delta w+v \cdot\left(\mathbf{C}_{u}-\mathbf{C}_{v}\right) .
\end{aligned}
$$

Now take the sum of (A.10)-(A.13). $\partial_{t}$ terms are

$$
\begin{aligned}
& \varphi u \cdot \partial_{t} w+w \cdot \partial_{t}(\varphi u)+v \cdot \partial_{t} w+w \cdot \partial_{t} v \\
& \quad=\partial_{t}(\varphi u \cdot w)+\partial_{t}(w \cdot v) \\
& \quad=\partial_{t}\left(|\varphi u|^{2}-|v|^{2}\right) .
\end{aligned}
$$

$\omega \times$ terms are

$$
w \cdot \omega \times \varphi u+\varphi u \cdot \omega \times w+w \cdot \omega \times v+v \cdot \omega \times w=0 .
$$


$\nabla \mathbf{R}$ terms are

$$
\begin{aligned}
& w \cdot \nabla \mathbf{R}(u \otimes \varphi u)+v \cdot \nabla \mathbf{R}(u \otimes w) \\
& \quad+\varphi u \cdot \nabla \mathbf{R}(u \otimes w)+w \cdot \nabla \mathbf{R}(u \otimes v) \\
& =\operatorname{div}(w \mathbf{R}(u \otimes \varphi u))+\operatorname{div}(v \mathbf{R}(u \otimes w)) \\
& \quad+\operatorname{div}(\varphi u \mathbf{R}(u \otimes w))+\operatorname{div}(w \mathbf{R}(u \otimes v)) \\
& \quad-\operatorname{div}(w) \nabla \mathbf{R}(u \otimes \varphi u)-\operatorname{div}(v) \nabla \mathbf{R}(u \otimes w) \\
& \quad-\operatorname{div}(\varphi u) \nabla \mathbf{R}(u \otimes w)-\operatorname{div}(\varphi) \nabla \mathbf{R}(u \otimes v) \\
& =2 \operatorname{div}(\varphi u \mathbf{R}(u \otimes \varphi u)-v \mathbf{R}(u \otimes v)) \\
& \quad-(u \cdot \nabla \varphi)(\nabla \mathbf{R}(u \otimes \varphi u)+\nabla \mathbf{R}(u \otimes w)+\nabla \mathbf{R}(u \otimes v)) \\
& =2 \operatorname{div}(\varphi u \mathbf{R}(u \otimes \varphi u)-v \mathbf{R}(u \otimes v))-2(u \cdot \nabla \varphi) \mathbf{R}(u \otimes \varphi u) .
\end{aligned}
$$

Here we use $\operatorname{div} v=0, \operatorname{div}(\varphi u)=\operatorname{div} w=u \cdot \nabla \varphi . \Delta$ terms are

$$
\begin{aligned}
\varphi u \cdot \Delta & w+w \cdot \Delta(\varphi u)+v \cdot \Delta w+w \cdot \Delta v \\
& =\Delta(u \cdot w)-2 \nabla(\varphi u): \nabla w+\Delta(v \cdot w)-2 \nabla v: \nabla w \\
& =\Delta\left(|\varphi u|^{2}-|v|^{2}\right)-2\left(|\nabla(\varphi u)|^{2}-|\nabla v|^{2}\right)
\end{aligned}
$$

Commutator terms are

$$
w \cdot \mathbf{C}_{u}+\varphi u \cdot\left(\mathbf{C}_{u}-\mathbf{C}_{v}\right)+w \cdot \mathbf{C}_{v}+v \cdot\left(\mathbf{C}_{u}-\mathbf{C}_{v}\right)=2 \varphi u \cdot \mathbf{C}_{u}-2 v \cdot \mathbf{C}_{v} .
$$

In summary, half the sum of these four identities A.10-A.13 gives

$$
\begin{gathered}
\partial_{t} \frac{|\varphi u|^{2}-|v|^{2}}{2}+\operatorname{div}(\varphi u \mathbf{R}(u \otimes \varphi u)-v \mathbf{R}(u \otimes v))+|\nabla(\varphi u)|^{2}-|\nabla v|^{2} \\
=\Delta \frac{|\varphi u|^{2}-|v|^{2}}{2}+\varphi u \cdot \mathbf{C}_{u}-v \cdot \mathbf{C}_{v}+(u \cdot \nabla \varphi) \mathbf{R}(\varphi u \otimes u) .
\end{gathered}
$$

Next, multiply local energy inequality of $u$ A.6. by $\varphi^{2}$,

$$
\begin{aligned}
\partial_{t} \frac{|\varphi u|^{2}}{2} & +|\varphi \nabla u|^{2}+\operatorname{div}\left(\varphi^{2} u \mathbf{R}(u \otimes u)\right) \\
\leq \Delta & \frac{|\varphi u|^{2}}{2}+\left[\varphi^{2}, \Delta\right] \frac{|u|^{2}}{2}+\left[\operatorname{div}, \varphi^{2}\right](u \mathbf{R}(u \otimes u)), \\
\partial_{t} \frac{|\varphi u|^{2}}{2}+ & |\nabla(\varphi u)|^{2}+\operatorname{div}(\varphi u \mathbf{R}(u \otimes \varphi u)) \\
\leq \Delta & \frac{|\varphi u|^{2}}{2}+\left[\varphi^{2}, \Delta\right] \frac{|u|^{2}}{2}+|u \otimes \nabla \varphi|^{2}+2(u \otimes \nabla \varphi):(\varphi \nabla u) \\
& +\left[\operatorname{div}, \varphi^{2}\right](u \mathbf{R}(u \otimes u))+\operatorname{div}(\varphi u[\mathbf{R}, \varphi](u \otimes u)) .
\end{aligned}
$$


The quadratic commutator terms in (A.15) are

$$
\begin{aligned}
{\left[\varphi^{2}, \Delta\right] } & \frac{|u|^{2}}{2}+|u \otimes \nabla \varphi|^{2}+2(u \otimes \nabla \varphi):(\varphi \nabla u) \\
& =\left[\varphi^{2}, \Delta\right] \frac{|u|^{2}}{2}+|u|^{2}|\nabla \varphi|^{2}+2 \nabla \varphi \cdot \varphi \nabla u \cdot u \\
& =-2 \nabla\left(\varphi^{2}\right) \cdot \nabla \frac{|u|^{2}}{2}-\Delta\left(\varphi^{2}\right) \frac{|u|^{2}}{2}+|u|^{2}|\nabla \varphi|^{2}+2 \nabla \varphi \cdot \nabla u \cdot \varphi u \\
& =-4 \varphi \nabla \varphi \cdot \nabla \frac{|u|^{2}}{2}-\frac{1}{2} \Delta\left(\varphi^{2}\right)|u|^{2}+|u|^{2}|\nabla \varphi|^{2}+2 \nabla \varphi \cdot \nabla u \cdot \varphi u \\
& =-2 \varphi \nabla \varphi \cdot \nabla u \cdot u-\varphi \Delta \varphi|u|^{2} \\
& =\varphi u \cdot(-2 \nabla \varphi \cdot \nabla u-(\Delta \varphi) u) \\
& =\varphi u \cdot[\varphi, \Delta] u .
\end{aligned}
$$

The cubic commutator terms in A.15) are

$$
\begin{aligned}
{\left[\operatorname{div}, \varphi^{2}\right] } & (u \mathbf{R}(u \otimes u))+\operatorname{div}(\varphi u[\mathbf{R}, \varphi](u \otimes u)) \\
= & 2 \varphi \nabla \varphi \cdot u \mathbf{R}(u \otimes u)+\varphi u \cdot \nabla[\mathbf{R}, \varphi](u \otimes u)+\operatorname{div}(\varphi u)[\mathbf{R}, \varphi](u \otimes u) \\
= & 2 \varphi(u \cdot \nabla \varphi) \mathbf{R}(u \otimes u)+\varphi u \cdot \nabla[\mathbf{R}, \varphi](u \otimes u)+(u \cdot \nabla \varphi)[\mathbf{R}, \varphi](u \otimes u) \\
= & 2 \varphi(u \cdot \nabla \varphi) \mathbf{R}(u \otimes u)+\varphi u \cdot \nabla[\mathbf{R}, \varphi](u \otimes u) \\
& \quad+(u \cdot \nabla \varphi) \mathbf{R}(\varphi u \otimes u)-(u \cdot \nabla \varphi) \varphi \mathbf{R}(u \otimes u) \\
= & \varphi u \cdot \nabla \varphi \mathbf{R}(u \otimes u)+\varphi u \cdot \nabla[\mathbf{R}, \varphi](u \otimes u)+(u \cdot \nabla \varphi) \mathbf{R}(\varphi u \otimes u) \\
= & \varphi u \cdot[\nabla, \varphi] \mathbf{R}(u \otimes u)+\varphi u \cdot \nabla[\mathbf{R}, \varphi](u \otimes u)+(u \cdot \nabla \varphi) \mathbf{R}(\varphi u \otimes u) \\
= & \varphi u \cdot([\nabla, \varphi] \mathbf{R}-\nabla[\varphi, \mathbf{R}])(u \otimes u)+(u \cdot \nabla \varphi) \mathbf{R}(\varphi u \otimes u) \\
= & \varphi u \cdot[\nabla \mathbf{R}, \varphi](u \otimes u)+(u \cdot \nabla \varphi) \mathbf{R}(\varphi u \otimes u) .
\end{aligned}
$$

Therefore, local energy inequality for $\varphi u$ can be simplified as

$$
\begin{aligned}
& \partial_{t} \frac{|\varphi u|^{2}}{2}+|\nabla(\varphi u)|^{2}+\operatorname{div}(\varphi u \mathbf{R}(u \otimes \varphi u)) \\
& \leq \Delta \frac{|\varphi u|^{2}}{2}+\varphi u \cdot \mathbf{C}_{u}+(u \cdot \nabla \varphi) \mathbf{R}(\varphi u \otimes u) .
\end{aligned}
$$

Subtracting (A.14) from this, we obtain (A.4).

\section{REFERENCES}

[BV19] Tristan Buckmaster and Vlad Vicol. Nonuniqueness of weak solutions to the NavierStokes equation. Ann. of Math. (2), 189(1):101-144, 2019.

[CF07] José A. Carrillo and Lucas C. F. Ferreira. Self-similar solutions and large time asymptotics for the dissipative quasi-geostrophic equation. Monatsh. Math., 151(2):111-142, 2007.

[CKN82] Luis Caffarelli, Robert Kohn, and Louis Nirenberg. Partial regularity of suitable weak solutions of the Navier-Stokes equations. Comm. Pure Appl. Math., 35(6):771-831, 1982.

[CLRM18] Diego Chamorro, Pierre-Gilles Lemarié-Rieusset, and Kawther Mayoufi. The role of the pressure in the partial regularity theory for weak solutions of the Navier-Stokes equations. Arch. Ration. Mech. Anal., 228(1):237-277, 2018.

[Con90] Peter Constantin. Navier-Stokes equations and area of interfaces. Comm. Math. Phys., 129(2):241-266, 1990. 
[CV14] Kyudong Choi and Alexis F. Vasseur. Estimates on fractional higher derivatives of weak solutions for the Navier-Stokes equations. Ann. Inst. H. Poincaré Anal. Non Linéaire, 31(5):899-945, 2014.

[FJR72] Eugene Fabes, B. Frank Jones, and Néstor Rivière. The initial value problem for the Navier-Stokes equations with data in $L^{p}$. Arch. Rational Mech. Anal., 45:222-240, 1972.

[Hop51] Eberhard Hopf. Über die Anfangswertaufgabe für die hydrodynamischen Grundgleichungen. Math. Nachr., 4:213-231, 1951.

[ISS03] Luis Iskauriaza, Gregory Serëgin, and Vladimír Shverak. $L_{3, \infty}$-solutions of NavierStokes equations and backward uniqueness. Uspekhi Mat. Nauk, 58(2(350)):3-44, 2003.

[KL57] A. A. Kiselev and Olga Ladyženskaya. On the existence and uniqueness of the solution of the nonstationary problem for a viscous, incompressible fluid. Izv. Akad. Nauk SSSR. Ser. Mat., 21:655-680, 1957.

[Ler34] Jean Leray. Sur le mouvement d'un liquide visqueux emplissant l'espace. Acta Math., 63(1):193-248, 1934.

[Lin98] Fanghua Lin. A new proof of the Caffarelli-Kohn-Nirenberg theorem. Comm. Pure Appl. Math., 51(3):241-257, 1998.

[Lio96] Pierre-Louis Lions. Mathematical topics in fluid mechanics. Vol. 1, volume 3 of Oxford Lecture Series in Mathematics and its Applications. The Clarendon Press, Oxford University Press, New York, 1996. Incompressible models, Oxford Science Publications.

[Pro59] Giovanni Prodi. Un teorema di unicità per le equazioni di Navier-Stokes. Ann. Mat. Pura Appl. (4), 48:173-182, 1959.

[Saw90] Eric Sawyer. Boundedness of classical operators on classical Lorentz spaces. Studia Math., 96(2):145-158, 1990.

[Sch76] Vladimir Scheffer. Partial regularity of solutions to the Navier-Stokes equations. Pacific J. Math., 66(2):535-552, 1976.

[Sch77] Vladimir Scheffer. Hausdorff measure and the Navier-Stokes equations. Comm. Math. Phys., 55(2):97-112, 1977.

[Sch78] Vladimir Scheffer. The Navier-Stokes equations in space dimension four. Comm. Math. Phys., 61(1):41-68, 1978.

[Sch80] Vladimir Scheffer. The Navier-Stokes equations on a bounded domain. Comm. Math. Phys., 73(1):1-42, 1980.

[Ser62] James Serrin. On the interior regularity of weak solutions of the Navier-Stokes equations. Arch. Rational Mech. Anal., 9:187-195, 1962.

[Ser63] James Serrin. The initial value problem for the Navier-Stokes equations. In Nonlinear Problems (Proc. Sympos., Madison, Wis., 1962), pages 69-98. Univ. of Wisconsin Press, Madison, Wis., 1963.

[Vas07] Alexis F. Vasseur. A new proof of partial regularity of solutions to Navier-Stokes equations. NoDEA Nonlinear Differential Equations Appl., 14(5-6):753-785, 2007.

[Vas10] Alexis Vasseur. Higher derivatives estimate for the 3D Navier-Stokes equation. Ann. Inst. H. Poincaré Anal. Non Linéaire, 27(5):1189-1204, 2010.

[Yan20] Jincheng Yang. Construction of Maximal Functions associated with Skewed Cylinders Generated by Incompressible Flows and Applications. arXiv e-prints, page arXiv:2008.05588, August 2020.

Department of Mathematics, The University of Texas at Austin, 2515 Speedway Stop C1200 Austin, TX 78712, USA

E-mail address: vasseur@math.utexas.edu

Department of Mathematics, The University of Texas at Austin, 2515 Speedway Stop C1200 Austin, TX 78712, USA

E-mail address: jcyang@math.utexas.edu 